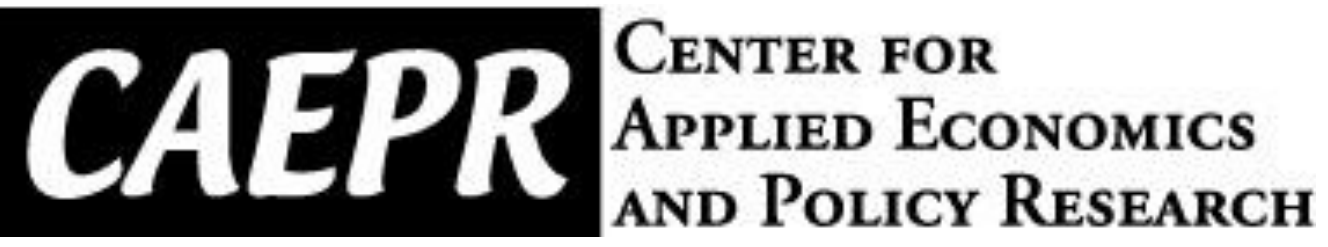

CAEPR Working Paper

\#2012-001

\title{
On existence, efficiency and bubbles of Ramsey equilibrium with borrowing constraints
}

\author{
Robert Becker \\ Indiana University \\ Stefano Bosi \\ EPEE, University of Eury \\ Cuong Le Van \\ CES, CNRS, VCREME and Hanoi WRU \\ Thomas Seegmuller \\ GREQAM, Aix-Marseille University
}

February 6, 2012

This paper can be downloaded without charge from the Social Science Research Network electronic library at: http://ssrn.com/abstract=2003000.

The Center for Applied Economics and Policy Research resides in the Department of Economics at Indiana University Bloomington. CAEPR can be found on the Internet at:

http://www.indiana.edu/ caepr. CAEPR can be reached via email at caepr@indiana.edu or via phone at 812-855-4050.

(C2012 by Robert Becker, Stefano Bosi, Cuong Le Van, and Thomas Seegmuller. All rights reserved. Short sections of text, not to exceed two paragraphs, may be quoted without explicit permission provided that full credit, including $₫$ notice, is given to the source. 


\title{
On existence, efficiency and bubbles of Ramsey equilibrium with borrowing constraints*
}

\author{
Robert Becker $†$ Stefano Bosi \\ Cuong Le $\operatorname{Van}^{\S}$ and Thomas Seegmuller $₫$
}

February 6, 2012

\begin{abstract}
We address the fundamental issues of existence and efficiency of a Ramsey equilibrium with heterogenous discounting, elastic labor supply and borrowing constraints.

In the first part, we prove the equilibrium existence in a truncated bounded economy through a fixed-point argument by Gale and Mas-Colell (1975). This equilibrium is also an equilibrium of any unbounded economy with the same fundamentals. The proof of existence is eventually given for an infinite-horizon economy as a limit of a sequence of truncated economies. Our general approach is suitable for applications to other models with different market imperfections.

In the second part, we show the impossibility of bubbles in a productive economy and we give sufficient conditions for equilibrium efficiency.

Keywords: Ramsey equilibrium, existence, efficiency, bubbles, heterogeneous agents, endogenous labor supply, borrowing constraint.

JEL classification: C62, D31, D91, G10.
\end{abstract}

\section{Introduction}

Frank Ramsey's (1928) seminal article on optimal capital accumulation is the fundamental model underlying much contemporary research in macroeconomic dynamics and growth theory. Although researchers modify the basic optimum

\footnotetext{
${ }^{*}$ This model has been presented to the international conference New Challenges for Macroeconomic Regulation held on June 2011 in Marseille. We would like to thank Monique Florenzano for her interesting comments.

$\dagger$ Department of Economics, Indiana University. E-mail: becker@indiana.edu.

${ }^{\ddagger} \mathrm{EPEE}$, University of Evry. E-mail: stefano.bosi@univ-evry.fr.

$\S$ CES, CNRS, VCREME and Hanoi WRU. E-mail: Cuong.Le-Van@univ-paris1.fr.

๑GREQAM, Aix-Marseille University. E-mail: Thomas.seegmuller@univ-amu.fr.
} 
growth model in many ways, it is common to assume an infinitely-lived representative agent whose preferences coincide with a central planner in economies for which the fundamental welfare theorems hold. Infinitely-lived representative agents also appear in models with various distortions even though a planning interpretation is no longer possible. Ramsey also formulated a conjecture about the long-run for an economy with several infinitely-lived agents, each maximizing a discounted sum of future utilities. His conjecture concerned the case in which each agent's pure rate of time preference differed. That is, some agents were more patient than others. His formal conjecture stated: ".. equilibrium would be attained by a division into two classes, the thrifty enjoying bliss and the improvident at the subsistence level" (Ramsey (1928), p. 559). This sentence ends the paper and means that, in the long run, the most patient agent(s) would hold all the capital, while the others would consume at the minimum level necessary to sustain their lives. Ramsey did not spell out the details of the equilibrium model that might give rise to this long-run solution, nor did he offer a notion of equilibrium for such an economy.

Becker (1980) proposed such a theory and demonstrated the Ramsey conjecture for that framework. Becker's model replaced subsistence by having workers earn a wage from the provision of their labor. A critical component of his model economy was a restriction placed on how much borrowing agents could undertake. This borrowing constraint binds for all but the most patient agent in the steady state solution. If this constraint had not been imposed, then a steady state might not even exist: for example, starting at Becker's steady state allocation, the relatively impatient households would borrow against the future stream of their labor incomes, consume more in the present and accept their consumption converges to zero as time tends to infinity even if rental and wage prices adjust. Thus, a binding borrowing constraint is critical for the existence of a steady state and it results in positive consumption equal to wage income at each time in the steady state for each agent more impatient than the most patient one. The agent with the lowest rate of time preference, or equivalently, the largest utility discount factor is defined as the most patient household. In the borrowing constrained steady state, that agent consumes a rental income from owning the economy's capital stock as well as its labor income. A complicating factor in dynamic analysis with this form of budget constraint occurs whenever an agent has zero capital at some time while also earning a positive wage income. Each agent always has the option to save and earn rental income in addition to labor income in the next period even if the agent has no capital. The relatively impatient agents rationally choose not to exercise this option in the steady state equilibrium. However, this might not by so away from the steady state allocation. In fact, this option is the source of many analytical complications as well as the richness of the dynamics found in subsequent work (referenced below) by Becker with his coauthors as well as the research published by Sorger, Bosi, and Seegmuller on this problem.

The many agent Ramsey equilibrium model with a borrowing constraint has incomplete markets as some intertemporal trades are excluded from the agents' opportunity sets by ruling out borrowing against the discounted value 
of future wage income. Models without this constraint permit all technically feasible intertemporal trades and are said to exhibit complete markets. The complete market case has been examined by many authors. The papers by Le Van and Vailakis (2003), Le Van et al. (2007), and Becker (2012) model complete markets and confirm the relatively impatient agents consumption tends to zero in the long-run within one-sector models. Le Van et al. (2007), which is pertinent for our paper, show this holds even when there is a labor-leisure choice. Earlier work along those lines in multi-sector models appears in Bewley (1982), Coles (1985, 1986), and for an exchange economy in Rader (1971, 1972, 1981) and Kehoe (1985).

The focus of our paper concerns the borrowing constrained Ramsey equilibrium model first present in Becker (1980) and developed over nearly thirty years in a series of papers included in our bibliography along with a survey paper published in 2006. Borrowing constraints constitute a credit market imperfection that has strong implications for the basic properties equilibria enjoy compared to the complete market case. In particular, there are three broad arenas in which the two models solutions differ in critical ways. The first concerns optimality. Credit market incompleteness entails the failure of the first welfare theorem. Even more strongly, it is now known from an example constructed by Becker et al. (2011) that a Ramsey equilibrium may be inefficient in the sense of Malinvaud (1953). As a matter of fact, it is no longer possible to prove the existence of a competitive equilibrium by studying the set of Pareto-efficient allocations using the Negishi (1960) approach as done by Le Van and Vailakis (2003) and Le Van et al. (2007), among others, in the absence of credit market imperfections. Also, see Kehoe et al. $(1989,1990)$ for related uses of the Negishi approach in many agent Ramsey models (with each agent possessing the same discount factor).

The second difference, already noted above, concerns stationary equilibrium properties. Impatient agents consume in the borrowing constrained stationary equilibrium. However, when these constraints are retired the steady state vanishes as shown in the complete markets counterpart by Le Van and Vailakis (2003) and Le Van et al. (2007). In fact, they show the equilibrium aggregate capital sequence converges to a particular capital stock, but this stock is not itself a steady state stock.

The third difference concerns dynamics of the aggregate equilibrium capital stock sequence. Representative agent optimal growth - perfect foresight equilibrium models generate optimal/equilibrium capital sequences which are monotonically convergent to a modified Golden-Rule steady state. This changes in presence of borrowing constraints. Persistent cycles can arise (Becker and Foias (1987, 1994), Sorger (1994)). Indeed, there can be chaotic solutions as well (Sorger (1995)). The source of these fluctuations lies in the possibility that aggregate capital income monotonicity can fail when the elasticity of substitution in the production functions is less than one. By way of contrast, when production is Cobb-Douglas, Becker and Foias (1987) show that equilibrium aggregate capital sequence is monotonic and converges to the steady state capital stock. 
The Ramsey conjecture holds under perfect competition with a borrowing constraint, which is one possible market imperfection. However, other forms of imperfections make the validity of the Ramsey conjecture fragile. Prominent examples are given by distortionary taxation and market power. Sarte (1997) and Sorger (2002) study a progressive capital income taxation, while Sorger $(2002,2005,2008)$ and Becker and Foias (2007) focus on the strategic interaction in the capital market. They prove the possibility of a long-run nondegenerate distribution of capital where (some) impatient agents hold capital. We focus attention in our paper entirely on the competitive case.

The closely related complete market models studied in Le Van and Vailakis (2003) and Le Van et al. (2007) also find that, under discounting heterogeneity, the monotonicity property of the representative agent counterpart does not carry over and that a twisted turnpike property holds instead (see Mitra (1979) and Becker (2012)). For example, the aggregate capital sequence can be eventually monotonic following an initial segment of damped oscillations. The very difference with the one-sector class of models à la Becker is that the optimal capital sequence always converges to a particular capital stock in the long run (which, paradoxically, is not a steady state equilibrium) and, thus, there is no room for persistent cycles.

Our paper addresses the fundamental theoretical question of the existence of an intertemporal equilibrium under borrowing constraints and heterogeneous households. Standard proofs for perfect and complete markets, such as the weighted welfare function Negishi (1960) technique, do not apply because markets are imperfect (for example, see Florenzano and Gourdel (1966) for a model with debt constraints). A different approach must be taken.

Becker et al. (1991) demonstrate the existence of an intertemporal equilibrium under borrowing constraints with inelastic labor supply. Their argument rests on the introduction of a tâtonnement map in which each fixed point yields an equilibrium. Specifically, they map the set of sequences of aggregate capital stocks to itself in such a way that a fixed point is an equilibrium. Their use of the aggregate capital stock sequences for this purpose allows them to formulate the map's domain as a compact (and convex) set in the product topology in the space of all nonnegative real sequences and thereby exploit an infinitedimensional fixed point argument to complete their demonstration. Their proof focuses on the interaction between agents' time preference and the productivity of the underlying one-sector technology. In doing so, they admit a wide-range of preferences over future consumption streams including ones with variable rates of time preference, including some that might not even by time-consistent as is the case with the more familiar time-additive and recursive utility representations. The sequence of one-period equilibrium rental rates can be calculated at the mapping's fixed point(s). This information can be used to test whether or not the Cass (1972) criterion for inefficiency in the sense of Malinvaud (1953) holds or not in equilibrium. However, there is nothing directly in their arguments to say whether or not the equilibrium found at a fixed point is efficient. Our approach, with some additional information, tells us whether an equilibrium path is efficient. 
The original Becker et al. (1991) existence proof does not settle the existence problem when agents have a labor-leisure choice. We address this problem. To do so, we specialize the intertemporal preferences to be additive separable across time and as well as during each period between consumption and leisure. Bosi and Seegmuller (2010) provide a local proof of existence of an intertemporal equilibrium with elastic labor supply in such a framework. Their argument rests on the existence of a local fixed point for the policy function based on the local stability properties of the steady state. Our paper supplies a considerably more general global existence proof for the elastic labor case than the local theorem reported them. For example, our result is not restricted to a neighborhood of the stationary state. We also permit capital to depreciate and thereby admit durable capital to the circulating capital framework in Becker et al. (1991).

There are two important by-products to our solution of the existence problem with elastic labor supply. First, our proof leads us to study the occurrence of asset price bubbles under agents' heterogeneity and market imperfections. Here, the present-value price of a unit of capital at each time defines a sequence of asset prices in our model. Second, we address whether or not the equilibrium is efficient by the Malinvaud (1953) criterion. As indicated above, one resolution of the efficiency question occurs when an additional property obtains that is analogous to one assumed by Becker and Mitra (2011) in the inelastic labor supply case. The way in which we rule out bubbly equilibria leads also links to another way to verify efficiency in Propositions 2 and 4 . Specifically, we prove that bubbles are ruled out under a sufficiently small capital depreciation rate and then show the equilibrium is efficient in this case. By way of contrast, under full depreciation, the equilibrium may be inefficient (see Becker et al. (2011)). The linkage between prospective bubbles, the rate of depreciation, and efficiency is a new insight compared to the work done by Becker and Mitra (2011) and Becker et al. (2011).

The fact that we can exclude asset pricing bubbles, at least in some important cases, is an interesting consequence of our resolution of the existence problem for this class of production economies with credit constraints. For instance, it is known that in models with only financial assets, there is room for asset price bubbles (see for instance Le Van and Vailakis (2012)). The novelty of our paper is that, under very mild assumptions, the introduction of a productive sector rules out the possibility of bubbles. In other words, investing in physical assets in an economy with borrowing constraints, but without money, can be one way of avoiding the emergence of rational speculative bubbles.

Becker et al. (1991) use infinite-dimensional commodity space techniques to prove an equilibrium exists. We solve the existence problem by taking a different route altogether. We begin by establishing an equilibrium exists for each possible finite-horizon economy. Florenzano (1999) provided a proof of equilibrium existence in a two-period incomplete market model by considering budget set correspondences and an auctioneer's correspondence, and using the Gale-Mas-Colell's (1975) fixed-point theorem. We introduce also a firm's correspondence and we apply the same idea to a truncated economy. Each of these economies, parameterized by the length of the horizon, is an approximation to 
the infinite horizon economy. By passing to the limit as time goes to infinity we find limiting sequences of prices and quantities which are verified to meet our equilibrium conditions. More precisely, the existence proof takes three steps:

- We first consider a time-truncated economy. Since the feasible allocations sets of our economy are uniformly bounded, we prove that there exists an equilibrium in a time-truncated bounded economy by Gale and Mas-Colell's (1975) theorem. Actually, this equilibrium turns out to be an equilibrium for the time-truncated economy as the uniform bounds are relaxed, as is commonly shown in finite-dimensional commodity space general equilibrium existence proofs.

- Second, we take the limit of a sequence of truncated, unbounded economies, and prove the existence of an intertemporal equilibrium in the limit economy.

- Third, we define an asset price bubble, provide conditions for a bubbleless equilibrium and conditions for equilibrium efficiency.

Most of the formal proofs are given in Appendices 1 to 4.

\section{The Ramsey model with heterogeneous house- holds and endogenous labor}

We present the basic framework we use. Time is discrete and the economy is perfectly competitive. There are two types of agents in the economy, firms and heterogeneous infinitely-lived households facing borrowing constraints. We clarify now the assumptions and the behavior of these two types of agents.

\section{$2.1 \quad$ Firms}

A representative firm produces a unique final good. The technology is represented by a constant returns to scale production function: $F\left(K_{t}, L_{t}\right)$, where $K_{t}$ and $L_{t}$ denote the demands for capital and labor. Profit maximization is correctly defined under the following assumption.

Assumption 1 The production function $F: \mathbb{R}_{+}^{2} \rightarrow \mathbb{R}_{+}$is $C^{1}$, homogeneous of degree one, strictly increasing and strictly concave. $\mathbb{R}_{+}$denotes the set of nonnegative real numbers. Inputs are essential: $F(0, L)=F(K, 0)=0$. Limit conditions for production and productivity hold: $F(K, L) \rightarrow+\infty$ either when $L>0$ and $K \rightarrow+\infty$ or when $K>0$ and $L \rightarrow+\infty$; in addition, $F_{L}(K, 0)=$ $+\infty$ for any $K>0$.

The price of the final good, the return on capital and the wage rate are denoted by $p_{t}, r_{t}$ and $w_{t}$ respectively. Profit maximization:

$$
\max _{K_{t}, L_{t}}\left[p_{t} F\left(K_{t}, L_{t}\right)-r_{t} K_{t}-w_{t} L_{t}\right]
$$


gives $\partial F / \partial K_{t}=r_{t} / p_{t}$ and $\partial F / \partial L_{t}=w_{t} / p_{t}$.

To simplify the proof of equilibrium existence, we introduce boundary conditions on capital productivity when the labor supply is maximal and equal to $m$.

Assumption $2\left(\partial F / \partial K_{t}\right)(0, m)>\delta$ and $\left(\partial F / \partial K_{t}\right)(+\infty, m)<\delta$, where $\delta \in$ $(0,1)$ denotes the rate of capital depreciation.

\subsection{Households}

We consider an economy without population growth where $m$ households work and consume. Each household $i$ is endowed with $k_{i 0}$ units of capital at period 0 and one unit of time per period. Leisure demand of agent $i$ at time $t$ is denoted by $\lambda_{i t}$ and the individual labor supply is given by $l_{i t}=1-\lambda_{i t}$. Individual wealth and consumption demand at time $t$ are denoted by $k_{i t}$ and $c_{i t}$.

Initial capital endowments are supposed to be positive.

Assumption $3 k_{i 0}>0$ for $i=1, \ldots, m$.

Each household maximizes a utility separable over time: $\sum_{t=0}^{T} \beta_{i}^{t} u_{i}\left(c_{i t}, \lambda_{i t}\right)$, where $\beta_{i} \in(0,1)$ is the discount factor of agent $i$.

Assumption $4 u_{i}: \mathbb{R}_{+}^{2} \rightarrow \mathbb{R}$ is $C^{0}$, strictly increasing and concave.

We observe a threefold heterogeneity: in terms of endowments $\left(k_{i 0}\right)$, discounting $\left(\beta_{i}\right)$ and per-period utility $\left(u_{i}\right)$.

In any period, the household faces a budget constraint:

$$
p_{t}\left[c_{i t}+k_{i t+1}-(1-\delta) k_{i t}\right] \leq r_{t} k_{i t}+w_{t}\left(1-\lambda_{i t}\right)
$$

It is known that, in economies with heterogenous discounting and no borrowing constraints, impatient agents borrow, consume more and work less in the short run, and that they consume less and work more in the long run to refund the debt to patient agents (see Le Van et al. (2007)). In our model, as in Becker (1980), agents are prevented from borrowing: $k_{i t} \geq 0$ for $i=1, \ldots, m$ and $t=1,2, \ldots$

\section{Definition of equilibrium}

We define an infinite-horizon sequences of prices and quantities:

$$
\left(\mathbf{p}, \mathbf{r}, \mathbf{w},\left(\mathbf{c}_{i}, \mathbf{k}_{i}, \boldsymbol{\lambda}_{i}\right)_{i=1}^{m}, \mathbf{K}, \mathbf{L}\right)
$$

where

$$
\begin{aligned}
(\mathbf{p}, \mathbf{r}, \mathbf{w}) & \equiv\left(\left(p_{t}\right)_{t=0}^{\infty},\left(r_{t}\right)_{t=0}^{\infty},\left(w_{t}\right)_{t=0}^{\infty}\right) \in \mathbb{R}^{\infty} \times \mathbb{R}_{+}^{\infty} \times \mathbb{R}_{+}^{\infty} \\
\left(\mathbf{c}_{i}, \mathbf{k}_{i}, \boldsymbol{\lambda}_{i}\right) & \equiv\left(\left(c_{i t}\right)_{t=0}^{\infty},\left(k_{i t}\right)_{t=1}^{\infty},\left(\lambda_{i t}\right)_{t=0}^{\infty}\right) \in \mathbb{R}_{+}^{\infty} \times \mathbb{R}^{\infty} \times \mathbb{R}_{+}^{\infty} \\
(\mathbf{K}, \mathbf{L}) & \equiv\left(\left(K_{t}\right)_{t=0}^{\infty},\left(L_{t}\right)_{t=0}^{\infty}\right) \in \mathbb{R}_{+}^{\infty} \times \mathbb{R}_{+}^{\infty}
\end{aligned}
$$

with $i=1, \ldots, m$. 
Definition 1 A Walrasian equilibrium $\left(\overline{\mathbf{p}}, \overline{\mathbf{r}}, \overline{\mathbf{w}},\left(\overline{\mathbf{c}}_{i}, \overline{\mathbf{k}}_{i}, \overline{\boldsymbol{\lambda}}_{i}\right)_{i=1}^{m}, \overline{\mathbf{K}}, \overline{\mathbf{L}}\right)$ satisfies the following conditions.

(1) Price positivity: $\bar{p}_{t}, \bar{r}_{t}, \bar{w}_{t}>0$ for $t=0,1, \ldots$

(2) Market clearing:

$$
\begin{aligned}
& \text { goods }: \sum_{i=1}^{m}\left[\bar{c}_{i t}+\bar{k}_{i t+1}-(1-\delta) \bar{k}_{i t}\right]=F\left(\bar{K}_{t}, \bar{L}_{t}\right) \\
& \text { capital }: \bar{K}_{t}=\sum_{i=1}^{m} \bar{k}_{i t} \\
& \text { labor : } \quad \bar{L}_{t}=\sum_{i=1}^{m} \bar{l}_{i t}
\end{aligned}
$$

for $t=0,1, \ldots$, where $l_{i t}=1-\lambda_{i t}$ denotes the individual labor supply.

(3) Optimal production plans: $\bar{p}_{t} F\left(\bar{K}_{t}, \bar{L}_{t}\right)-\bar{r}_{t} \bar{K}_{t}-\bar{w}_{t} \bar{L}_{t}$ is the value of the program: $\max \left[\bar{p}_{t} F\left(K_{t}, L_{t}\right)-\bar{r}_{t} K_{t}-\bar{w}_{t} L_{t}\right]$, under the constraints $K_{t} \geq 0$ and $L_{t} \geq 0$ for $t=0,1, \ldots$

(4) Optimal consumption plans: $\sum_{t=0}^{\infty} \beta_{i}^{t} u_{i}\left(\bar{c}_{i t}, \bar{\lambda}_{i t}\right)$ is the value of the program: $\max \sum_{t=0}^{\infty} \beta_{i}^{t} u_{i}\left(c_{i t}, \lambda_{i t}\right)$, under the following constraints:

$$
\begin{aligned}
& \text { budget constraint : } \quad \bar{p}_{t}\left[c_{i t}+k_{i t+1}-(1-\delta) k_{i t}\right] \leq \bar{r}_{t} k_{i t}+\bar{w}_{t}\left(1-\lambda_{i t}\right) \\
& \text { borrowing constraint : } k_{i t+1} \geq 0 \\
& \text { leisure endowment : } 0 \leq \lambda_{i t} \leq 1 \\
& \text { capital endowment : } k_{i 0} \geq 0 \text { given }
\end{aligned}
$$

for $t=0,1, \ldots$

The following claims are essential in our paper.

Claim 1 Labor supply is uniformly bounded.

Proof. At the individual level, because $l_{i t}=1-\lambda_{i t} \in[0,1]$. At the aggregate level, because $0 \leq \sum_{i=1}^{m} l_{i t} \leq m$.

The following claim is fundamental to prove the existence of an equilibrium in a finite-horizon economy. The argument mainly rests on the concavity of production function (Assumption 1) and the boundary conditions on capital productivity (Assumption 2). It ensures that capital is uniformly bounded as well as the other equilibrium components (consumption boundedness follows at the end of the section).

Claim 2 Under Assumptions 1 and 2, individual and aggregate capital supplies are uniformly bounded.

Proof. At the individual level, because of the borrowing constraint, we have $0 \leq k_{i t} \leq \sum_{h=1}^{m} k_{h t}$. 
To prove that the individual capital supply is uniformly bounded, we prove that the aggregate capital supply is uniformly bounded. We want to show that $0 \leq \sum_{h=1}^{m} k_{h t} \leq \max \left\{x, \sum_{i=1}^{m} k_{i 0}\right\} \equiv A$, where $x$ is the unique solution of

$$
x=(1-\delta) x+F(x, m)
$$

Since $F$ is $C^{1}$, increasing and concave, $F(0, L)=0$ and $1-\delta+\left(\partial F / \partial K_{t}\right)(0, m)>$ $1>1-\delta+\left(\partial F / \partial K_{t}\right)(+\infty, m)$ (Assumptions 1 and 2), the nonzero solution of (1) is unique. Moreover, $x \leq y$ implies

$$
(1-\delta) y+F(y, m) \leq y
$$

We notice that

$$
\begin{aligned}
\sum_{i=1}^{m} k_{i t+1} & \leq \sum_{i=1}^{m}\left(c_{i t}+k_{i t+1}\right) \leq(1-\delta) \sum_{i=1}^{m} k_{i t}+F\left(\sum_{i=1}^{m} k_{i t}, \sum_{i=1}^{m} l_{i t}\right) \\
& \leq(1-\delta) \sum_{i=1}^{m} k_{i t}+F\left(\sum_{i=1}^{m} k_{i t}, m\right)
\end{aligned}
$$

because $F$ is increasing, the capital employed cannot exceed its aggregate supply $\sum_{i=1}^{m} k_{i t}$ and $\sum_{i=1}^{m} l_{i t} \leq m$. Let $x_{t} \equiv \sum_{i=1}^{m} k_{i t}$. Then, $x_{t+1} \leq(1-\delta) x_{t}+$ $F\left(x_{t}, m\right)$.

We observe that $x_{0} \leq \max \left\{x, x_{0}\right\} \equiv A$. Therefore, $x_{1} \leq(1-\delta) x_{0}+$ $F\left(x_{0}, m\right) \leq(1-\delta) A+F(A, m) \leq A$ because $x \leq A$ and, from (2), (1b) $A+F(A, m) \leq A$. Iterating the argument, we find $x_{t} \leq A$ for $t=0,1, \ldots$

Claim 3 Under Assumptions 1 and 2, individual and aggregate consumption demands are uniformly bounded.

Proof. At the individual level, we have $0 \leq c_{i t} \leq \sum_{h=1}^{m} c_{h t}$.

To prove that the individual consumption is uniformly bounded, we prove that the aggregate consumption is uniformly bounded.

$$
\begin{aligned}
\sum_{i=1}^{m} c_{i t} & \leq \sum_{i=1}^{m}\left(c_{i t}+k_{i t+1}\right) \leq(1-\delta) \sum_{i=1}^{m} k_{i t}+F\left(\sum_{i=1}^{m} k_{i t}, m\right) \\
& \leq(1-\delta) A+F(A, m) \leq A
\end{aligned}
$$

\section{On the existence of equilibrium in a finite- horizon economy}

We consider an economy which goes on for $T+1$ periods: $t=0, \ldots, T$. 
Focus first on a bounded economy, that is choose sufficiently large bounds for quantities:

$$
\begin{aligned}
X_{i} & \equiv\left\{\left(c_{i 0}, \ldots, c_{i T}\right): 0 \leq c_{i t} \leq B_{c}\right\}=\left[0, B_{c}\right]^{T+1} \text { with } A<B_{c} \\
Y_{i} & \equiv\left\{\left(k_{i 1}, \ldots, k_{i T}\right): 0 \leq k_{i t} \leq B_{k}\right\}=\left[0, B_{k}\right]^{T} \text { with } A<B_{k} \\
Z_{i} & \equiv\left\{\left(\lambda_{i 0}, \ldots, \lambda_{i T}\right): 0 \leq \lambda_{i t} \leq 1\right\}=[0,1]^{T+1} \\
Y & \equiv\left\{\left(K_{0}, \ldots, K_{T}\right): 0 \leq K_{t} \leq B_{K}\right\}=\left[0, B_{K}\right]^{T+1} \text { with } A<B_{K} \\
Z & \equiv\left\{\left(L_{0}, \ldots, L_{T}\right): 0 \leq L_{t} \leq B_{L}\right\}=\left[0, B_{L}\right]^{T+1} \text { with } m<B_{L}
\end{aligned}
$$

with $m B_{k}<B_{K}$.

We notice that $k_{i 0}$ is given and that the borrowing constraints (inequalities $k_{i t} \geq 0$ ) capture the imperfection in the credit market. ${ }^{1}$

Let $\mathcal{E}^{T}$ denote this economy with technology and preferences as in Assumptions 1 to 4 . Let $X_{i}, Y_{i}$ and $Z_{i}$ be the $i$ th consumer-worker's bounded sets for consumption demand, capital supply and leisure demand respectively $(i=1, \ldots, m)$. Eventually, let $Y$ and $Z$ be the firm's bounded sets for capital and labor demands.

Proposition 1 Under the Assumptions 1, 2, 3 and 4, there exists an equilibrium $\left(\overline{\mathbf{p}}, \overline{\mathbf{r}}, \overline{\mathbf{w}},\left(\overline{\mathbf{c}}_{h}, \overline{\mathbf{k}}_{h}, \overline{\boldsymbol{\lambda}}_{h}\right)_{h=1}^{m}, \overline{\mathbf{K}}, \overline{\mathbf{L}}\right)$ for the finite-horizon bounded economy $\mathcal{E}^{T}$.

Proof. The proof is articulated in many claims and given in Appendix 1.

Focus now on an unbounded economy.

Theorem 4 Any equilibrium of $\mathcal{E}^{T}$ is an equilibrium for the finite-horizon unbounded economy.

Proof. Let $\left(\overline{\mathbf{p}}, \overline{\mathbf{r}}, \overline{\mathbf{w}},\left(\overline{\mathbf{c}}_{h}, \overline{\mathbf{k}}_{h}, \overline{\boldsymbol{\lambda}}_{h}\right)_{h=1}^{m}, \overline{\mathbf{K}}, \overline{\mathbf{L}}\right)$ with $\bar{p}_{t}, \bar{r}_{t}, \bar{w}_{t}>0, t=0, \ldots, T$, be an equilibrium of $\mathcal{E}^{T}$

Let $\left(\mathbf{c}_{i}, \mathbf{k}_{i}, \boldsymbol{\lambda}_{i}\right)$ verify $\sum_{t=0}^{T} \beta_{i}^{t} u_{i}\left(c_{i t}, \lambda_{i t}\right)>\sum_{t=0}^{T} \beta_{i}^{t} u_{i}\left(\bar{c}_{i t}, \bar{\lambda}_{i t}\right)$. We want to prove that this allocation violates at least one budget constraint, that is that there exists $t$ such that

$$
\bar{p}_{t}\left[c_{i t}+k_{i t+1}-(1-\delta) k_{i t}\right]>\bar{r}_{t} k_{i t}+\bar{w}_{t}\left(1-\lambda_{i t}\right)
$$

Focus on a strictly convex combination of $\left(\mathbf{c}_{i}, \mathbf{k}_{i}, \boldsymbol{\lambda}_{i}\right)$ and $\left(\overline{\mathbf{c}}_{i}, \overline{\mathbf{k}}_{i}, \overline{\boldsymbol{\lambda}}_{i}\right)$ :

$$
\begin{aligned}
c_{i t}(\gamma) & \equiv \gamma c_{i t}+(1-\gamma) \bar{c}_{i t} \\
k_{i t}(\gamma) & \equiv \gamma k_{i t}+(1-\gamma) \bar{k}_{i t} \\
\lambda_{i t}(\gamma) & \equiv \gamma \lambda_{i t}+(1-\gamma) \bar{\lambda}_{i t}
\end{aligned}
$$

with $0<\gamma<1$. Notice that we assume that the bounds satisfy $B_{c}, B_{k}, B_{K}>A$ and $B_{L}>m$ in order ensure that we enter the bounded economy when the parameter $\gamma$ is sufficiently close to 0 .

\footnotetext{
${ }^{1}$ A possible generalization of credit constraints is $h_{i} \leq k_{i t}$ with $h_{i}<0$ given.
} 
Entering the bounded economy means $\left(\mathbf{c}_{i}(\gamma), \mathbf{k}_{i}(\gamma), \boldsymbol{\lambda}_{i}(\gamma)\right) \in X_{i} \times Y_{i} \times Z_{i}$. In this case, because of the concavity of the utility function, we find

$$
\begin{aligned}
\sum_{t=0}^{T} \beta_{i}^{t} u_{i}\left(c_{i t}(\gamma), \lambda_{i t}(\gamma)\right) & \geq \gamma \sum_{t=0}^{T} \beta_{i}^{t} u_{i}\left(c_{i t}, \lambda_{i t}\right)+(1-\gamma) \sum_{t=0}^{T} \beta_{i}^{t} u_{i}\left(\bar{c}_{i t}, \bar{\lambda}_{i t}\right) \\
& >\sum_{t=0}^{T} \beta_{i}^{t} u_{i}\left(\bar{c}_{i t}, \bar{\lambda}_{i t}\right)
\end{aligned}
$$

Since $\left(\mathbf{c}_{i}(\gamma), \mathbf{k}_{i}(\gamma), \boldsymbol{\lambda}_{i}(\gamma)\right) \in X_{i} \times Y_{i} \times Z_{i}$ and $\left(\overline{\mathbf{p}}, \overline{\mathbf{r}}, \overline{\mathbf{w}},\left(\overline{\mathbf{c}}_{h}, \overline{\mathbf{k}}_{h}, \overline{\boldsymbol{\lambda}}_{h}\right)_{h=1}^{m}, \overline{\mathbf{K}}, \overline{\mathbf{L}}\right)$ is an equilibrium for this economy, there exists $t \in\{0, \ldots, T\}$ such that

$$
\bar{p}_{t}\left[c_{i t}(\gamma)+k_{i t+1}(\gamma)-(1-\delta) k_{i t}(\gamma)\right]>\bar{r}_{t} k_{i t}(\gamma)+\bar{w}_{t}\left(1-\lambda_{i t}(\gamma)\right)
$$

Replacing (4), we get

$$
\begin{aligned}
& \bar{p}_{t}\left(\gamma c_{i t}+(1-\gamma) \bar{c}_{i t}+\gamma k_{i t+1}+(1-\gamma) \bar{k}_{i t+1}-(1-\delta)\left[\gamma k_{i t}+(1-\gamma) \bar{k}_{i t}\right]\right) \\
> & \bar{r}_{t}\left[\gamma k_{i t}+(1-\gamma) \bar{k}_{i t}\right]+\bar{w}_{t}\left(1-\left[\gamma \lambda_{i t}+(1-\gamma) \bar{\lambda}_{i t}\right]\right)
\end{aligned}
$$

that is

$$
\begin{aligned}
\gamma \bar{p}_{t}\left[c_{i t}+k_{i t+1}-(1-\delta) k_{i t}\right]+(1-\gamma) \bar{p}_{t}\left[\bar{c}_{i t}+\bar{k}_{i t+1}-(1-\delta) \bar{k}_{i t}\right] \\
>\gamma\left[\bar{r}_{t} k_{i t}+\bar{w}_{t}\left(1-\lambda_{i t}\right)\right]+(1-\gamma)\left[\bar{r}_{t} \bar{k}_{i t}+\bar{w}_{t}\left(1-\bar{\lambda}_{i t}\right)\right]
\end{aligned}
$$

Since $\bar{p}_{t}\left[\bar{c}_{i t}+\bar{k}_{i t+1}-(1-\delta) \bar{k}_{i t}\right]=\bar{r}_{t} \bar{k}_{i t}+\bar{w}_{t}\left(1-\bar{\lambda}_{i t}\right)$, we obtain (3). Thus $\left(\overline{\mathbf{p}}, \overline{\mathbf{r}}, \overline{\mathbf{w}},\left(\overline{\mathbf{c}}_{h}, \overline{\mathbf{k}}_{h}, \overline{\boldsymbol{\lambda}}_{h}\right)_{h=1}^{m}, \overline{\mathbf{K}}, \overline{\mathbf{L}}\right)$ is also an equilibrium for the unbounded economy.

\section{On the existence of equilibrium in an infinite- horizon economy}

In the following, we need more structure and, namely, a separable utility. Let us denote by $u_{i}$ and $v_{i}$ the utilities of consumption and leisure, and by $w_{i}$ their sum: $w_{i}\left(c_{i t}, \lambda_{i t}\right) \equiv u_{i}\left(c_{i t}\right)+v_{i}\left(\lambda_{i t}\right)$. The next assumption replaces Assumption 4 .

Assumption 5 The utility function is separable: $w_{i}\left(c_{i t}, \lambda_{i t}\right) \equiv u_{i}\left(c_{i t}\right)+v_{i}\left(\lambda_{i t}\right)$, with $u_{i}, v_{i}: \mathbb{R}_{+} \rightarrow \mathbb{R}$ and $u_{i}, v_{i} \in C^{1}$. In addition, we require that $u_{i}(0)=$ $v_{i}(0)=0, u_{i}^{\prime}(0)=v_{i}^{\prime}(0)=+\infty, u_{i}^{\prime}\left(c_{i t}\right), v_{i}^{\prime}\left(\lambda_{i t}\right)>0$ for $c_{i t}, \lambda_{i t}>0$, and that functions $u, v$ are concave.

Theorem 5 Under the Assumptions 1, 2, 3 and 5, there exists an equilibrium in the infinite-horizon economy with endogenous labor supply and borrowing constraints.

Proof. We consider a sequence of time-truncated economies and the associated equilibria. We prove that there exists a sequence of equilibria which converges, when the horizon $T$ goes to infinity, to an equilibrium of the infinite-horizon economy. The proof is detailed in Appendix 2. 


\section{Bubbles}

There is considerable interest in whether or not a perfect foresight equilibrium capital asset price sequence is consistent with the notion of a rational pricing bubble. Tirole (1990), for example, argues bubbles can arise if there are multiple solutions of the forward iterates of the no-arbitrage relation for a perfect foresight equilibrium. He goes on to show the possibility that the shadow prices supporting an efficient allocation in the sense of Malinvaud (1953) can exhibit a bubble. Under some supplementary conditions, developed below and in the following section, we find a linkage between ruling out bubbles and proving efficiency of an equilibrium.

Let $\left(\overline{\mathbf{p}}, \overline{\mathbf{r}}, \overline{\mathbf{w}},\left(\overline{\mathbf{c}}_{i}, \overline{\mathbf{k}}_{i}, \overline{\boldsymbol{\lambda}}_{i}\right)_{i=1}^{m}, \overline{\mathbf{K}}, \overline{\mathbf{L}}\right)$ denote an equilibrium.

Claim 6 For any individual $i$, the equilibrium sequence of multipliers $\overline{\boldsymbol{\mu}}_{i} \equiv$ $\left(\bar{\mu}_{i t}\right)_{t=0}^{\infty}$ exists. The first-order conditions:

$$
\beta_{i}^{t} u_{i}^{\prime}\left(\bar{c}_{i t}^{T}\right)=\bar{\mu}_{i t}^{T} \bar{p}_{t}^{T} \geq \bar{\mu}_{i t+1}^{T} \bar{p}_{t+1}^{T}(1-\delta)+\bar{\mu}_{i t+1}^{T} \bar{r}_{t+1}^{T}
$$

for $i=1, \ldots, m, t=0, \ldots, T$, with equality when $\bar{k}_{i t+1}^{T}>0$ (point (7) in Claim 15), are satisfied in the limit economy.

Proof. The proof is given in Appendix 4.

Let us introduce a ratio of multiplier values:

$$
q_{t+1} \equiv \max _{i} \frac{\mu_{i t+1} p_{t+1}}{\mu_{i t} p_{t}}
$$

Since $\bar{K}_{t}>0$ for any $t$, there exists $i$ such that $\bar{k}_{i t+1}>0$ and $\bar{\mu}_{i t} \bar{p}_{t}=$ $\bar{\mu}_{i t+1}\left[\bar{p}_{t+1}(1-\delta)+\bar{r}_{t+1}\right]$. We observe that

$$
\frac{\bar{\mu}_{i t+1} \bar{p}_{t+1}}{\bar{\mu}_{i t} \bar{p}_{t}} \leq \frac{1}{1-\delta+\bar{\rho}_{t+1}} \text { and } \frac{\bar{\mu}_{i t+1} \bar{p}_{t+1}}{\bar{\mu}_{i t} \bar{p}_{t}}=\frac{1}{1-\delta+\bar{\rho}_{t+1}} \text { if } \bar{k}_{i t+1}>0
$$

where the real return on capital is defined by $\rho_{t} \equiv r_{t} / p_{t}$.

Thus, the equilibrium ratio $\bar{q}_{t}$ has a natural interpretation as a market discount factor:

$$
\bar{q}_{t+1}=\max _{i} \frac{\beta_{i} u_{i}^{\prime}\left(\bar{c}_{i t+1}\right)}{u_{i}^{\prime}\left(\bar{c}_{i t}\right)}=\frac{1}{1-\delta+\bar{\rho}_{t+1}}
$$

Let

$$
\begin{aligned}
Q_{0} & \equiv 1 \\
Q_{t} & \equiv \prod_{s=1}^{t} q_{s} \text { for } t>0
\end{aligned}
$$

Clearly, $\bar{Q}_{0}=1$ and $\bar{Q}_{t}=\prod_{s=1}^{t}\left(1-\delta+\bar{\rho}_{s}\right)^{-1}$ for $t>0 . \bar{Q}_{t}$ is the present value of a unit of capital of period $t$. For any $t$, we obtain:

$$
\bar{Q}_{t}=\bar{Q}_{t+1}\left(1-\delta+\bar{\rho}_{t+1}\right)
$$

and, by induction, $1=\bar{Q}_{0}=\bar{Q}_{T}(1-\delta)^{T}+\sum_{t=1}^{T} \bar{Q}_{t} \bar{\rho}_{t}(1-\delta)^{t-1}$. 
Definition 2 We define the fundamental value of capital as

$$
\bar{v}_{0} \equiv \sum_{t=1}^{+\infty} \bar{\rho}_{t} \bar{Q}_{t}(1-\delta)^{t-1}
$$

The economy is said to experience a bubble if $\lim _{T \rightarrow+\infty} \bar{Q}_{T}(1-\delta)^{T}>0$. Otherwise $\left(\lim _{T \rightarrow+\infty} \bar{Q}_{T}(1-\delta)^{T}=0\right)$, there is no bubble.

If $i=1$ denotes the most patient agent with $\beta_{i}<\beta_{1}$ for $i=2, \ldots, m$, at the stationary equilibrium, $\bar{Q}_{t}$ coincides with the discount factor of the patient agent, the only one with $\bar{k}_{i t}>0$ in the long run:

$$
\bar{Q}_{t} \equiv \prod_{s=1}^{t} \bar{q}_{s}=\prod_{s=1}^{t} \frac{\beta_{1} u_{1}^{\prime}\left(\bar{c}_{1 s+1}\right)}{u_{1}^{\prime}\left(\bar{c}_{1 s}\right)}=\prod_{s=1}^{t} \beta_{1}=\beta_{1}^{t}
$$

Focus now on the crucial question of existence of bubbles in a productive economy.

We know that a bubble exists if $\lim _{T \rightarrow+\infty} \bar{Q}_{T}(1-\delta)^{T}>0$. We have

$$
\bar{Q}_{T}(1-\delta)^{T}=\prod_{s=1}^{T} \frac{1-\delta}{1-\delta+\bar{\rho}_{s}}
$$

Let us show that a productive economy experiences no bubbles. The proof rests on the following lemma.

Lemma 1 If the economy experiences a bubble, then $\bar{\rho}_{t}$ converges to zero.

Proof. It is equivalent to prove that, if $\bar{\rho}_{t}$ does not converge to zero, there are no bubbles. If $\bar{\rho}_{t}$ does not converge, there are, equivalently, $\varepsilon>0$ and an infinite and increasing sequence $\left(t_{i}\right)_{i=1}^{+\infty}$ such that $\bar{\rho}_{t_{i}} \geq \varepsilon$ for all $i=1,2, \ldots$ For $T>t_{n}$, we get

$$
\bar{Q}_{T}(1-\delta)^{T}=\prod_{s=1}^{T} \frac{1-\delta}{1-\delta+\bar{\rho}_{s}} \leq \prod_{i=1}^{n} \frac{1-\delta}{1-\delta+\bar{\rho}_{t_{i}}} \leq\left(\frac{1-\delta}{1-\delta+\varepsilon}\right)^{n}
$$

and

$$
0 \leq \lim \sup _{T \rightarrow+\infty} \bar{Q}_{T}(1-\delta)^{T} \leq \lim _{n \rightarrow+\infty}\left(\frac{1-\delta}{1-\delta+\varepsilon}\right)^{n}=0
$$

Lemma 2 Let $\psi$ be a concave, continuous, strictly increasing function on $\mathbb{R}_{+}$, differentiable in $\mathbb{R}_{++}$. Then, $\lim _{x \rightarrow 0_{+}} \psi^{\prime}(x)>0$.

Proof. We have always $0<\psi^{\prime}(x) \leq \lim _{x \rightarrow 0_{+}} \psi^{\prime}(x)$, for all $x>0$. If $\lim _{x \rightarrow 0_{+}} \psi^{\prime}(x)=0$, then $\psi^{\prime}(x)=0$ for every $x>0$ against the increasingness of $\psi$.

Now, we are able to prove the main theorem. 
Theorem 7 Our productive economy experiences no bubble.

Proof. First, observe that the production function $F$ satisfies Assumption 1. Second, from Lemma 2, we have

$$
\lim _{b \rightarrow 0_{+}} F_{L}(1, b)>0
$$

Since $F$ is homogeneous of degree one, we have, for $K>0$ and $L>0$, $F_{K}(K, L)=F_{K}(K / L, 1)$ and $F_{L}(K, L)=F_{L}(1, L / K)$. Let $\left(\bar{K}_{t}, \bar{L}_{t}\right)$ be an equilibrium sequence of aggregate capital stocks and labors. Observe that $\bar{\rho}_{t}=$ $F_{K}\left(\bar{K}_{t} / \bar{L}_{t}, 1\right)$. Since $F$ is differentiable and concave, we have for any $t$

$$
\bar{\rho}_{t} \geq \lim _{a \rightarrow+\infty} F_{K}(a, 1)
$$

Suppose the economy has a bubble in prices. Then, from Lemma $1, \bar{\rho}_{t}$ converges to zero. But from (9), $\bar{K}_{t} / \bar{L}_{t}$ tends to infinity, or equivalently, $\bar{L}_{t} / \bar{K}_{t}$ goes to 0 . Since $\bar{K}_{t}$ is positive and bounded above (see Claim 2), we obtain $\bar{L}_{t} \rightarrow 0$. Recall that

$$
\bar{C}_{t}+\bar{K}_{t+1}=F\left(\bar{K}_{t}, \bar{L}_{t}\right)+(1-\delta) \bar{K}_{t}=\bar{K}_{t}\left[F\left(1, \bar{L}_{t} / \bar{K}_{t}\right)+1-\delta\right]
$$

and choose $\varepsilon>0$ such that $F(1, \varepsilon)+1-\delta<1$. There exists $T$ such that for any $t>T, K_{t+1} \leq \bar{K}_{t}\left[F\left(1, \bar{L}_{t} / \bar{K}_{t}\right)+1-\delta\right]<[F(1, \varepsilon)+1-\delta] \bar{K}_{t}$. This implies $\bar{K}_{t} \rightarrow 0$ when $t$ tends to infinity, and $\bar{C}_{t} \rightarrow 0$ too.

Reconsider the first-order conditions of point 8 in Claim 15:

$$
\bar{\omega}_{t} u_{i}^{\prime}\left(\bar{c}_{i t}\right) \leq v_{i}^{\prime}\left(1-\bar{l}_{i t}\right)
$$

We know that $\bar{\omega}_{t}=F_{L}\left(\bar{K}_{t}, \bar{L}_{t}\right)$ (see point (3) in Claim 15). Since $\bar{L}_{t} / \bar{K}_{t}$ converges to 0 , according to (8), we have $\lim _{t \rightarrow+\infty} \bar{\omega}_{t}=\lim _{t \rightarrow+\infty} F_{L}\left(1, \bar{L}_{t} / \bar{K}_{t}\right)>$ 0. $\lim _{t \rightarrow+\infty} \bar{C}_{t}=0$ implies $\lim _{t \rightarrow+\infty} \bar{c}_{i t}=0$ and $\lim _{t \rightarrow+\infty} u_{i}^{\prime}\left(\bar{c}_{i t}\right)=+\infty$ for any $i$. Thus, $\lim _{t \rightarrow+\infty}\left[\bar{\omega}_{t} u_{i}^{\prime}\left(\bar{c}_{i t}\right)\right]=+\infty$ on the left-hand side of (10). We notice also that $\bar{L}_{t} \rightarrow 0$ implies $\bar{l}_{i t} \rightarrow 0$ for any $i$, and, so, $\lim _{t \rightarrow+\infty} v_{i}^{\prime}\left(1-\bar{l}_{i t}\right)=v_{i}^{\prime}(1)<$ $+\infty$ on the right-hand side of (10), that is a contradiction. In other terms, there is no room for bubbles in our economy.

\section{Efficiency}

As above, $\left(\overline{\mathbf{p}}, \overline{\mathbf{r}}, \overline{\mathbf{w}},\left(\overline{\mathbf{c}}_{i}, \overline{\mathbf{k}}_{i}, \overline{\boldsymbol{\lambda}}_{i}\right)_{i=1}^{m}, \overline{\mathbf{K}}, \overline{\mathbf{L}}\right)$ denotes an equilibrium. Following Malinvaud (1953) and Becker and Mitra (2011), we introduce the following definition.

Definition 3 An equilibrium is efficient if there is no sequence of total consumption, capital and labor $\left(C_{t}, K_{t}, L_{t}\right)$ which satisfies, for $t=0,1, \ldots$

$$
C_{t}+K_{t+1}-(1-\delta) K_{t}=F\left(K_{t}, L_{t}\right)
$$

(feasibility) with $C_{t} \geq \bar{C}_{t}$ and $m-L_{t} \geq m-\bar{L}_{t}$ for $t=0,1, \ldots$ with at least one strict inequality for consumption or for leisure. $K_{0}$, the aggregate capital endowment, is given. 
Proposition 2 The present value of one unit of capital $\bar{Q}_{t}$ is defined by (6) and (5). If $\lim _{t \rightarrow \infty} \bar{Q}_{t}=0$, the equilibrium is efficient.

Proof. The proof is given in Appendix 4.

It is worthy to notice that (1) the lack of bubbles in a productive economy means $\lim _{t \rightarrow+\infty} \bar{Q}_{t}(1-\delta)^{t}=0$ which does not imply $\lim _{t \rightarrow \infty} \bar{Q}_{t}=0$, and, actually, (2) for the proof (see Appendix 4), the sufficient condition for efficiency is $\lim _{t \rightarrow \infty} \bar{Q}_{t} \bar{K}_{t+1}=0$ which is the condition given by Malinvaud (1953). This condition is equivalent, in our model, to $\lim _{t \rightarrow \infty} \bar{Q}_{t}=0$ since the sequence $\left(K_{t}\right)$ is uniformly bounded.

Let us show that (1), when the depreciation rate $\delta>F_{K}(\infty, 1)$ (Assumption 2 ) is sufficiently close to $F_{K}(\infty, 1)$, any equilibrium is efficient, and (2) an equilibrium is efficient if a property holds that, for some agent $i$, the sequences of her/his consumptions $\left(c_{i t}\right)$ and capital stocks $\left(k_{i t}\right)$ are uniformly bounded away from zero for $t$ large enough.

According to Assumption $1, F_{L}(1,0)=+\infty$. Define $x(\alpha)$ by $F_{K}(x(\alpha), 1)=$ $\alpha$ for any $\alpha \in\left(F_{K}(\infty, 1), F_{K}(0,1)\right)$. The function $x$ is continuous and decreasing. If $\alpha \rightarrow F_{K}(\infty, 1)$, then $x(\alpha) \rightarrow+\infty$ and $F_{L}(1,1 / x(\alpha)) \rightarrow+\infty$. Let $M$ denote the bound of the paths of capital stocks $\left(K_{t}\right)$ and total consumptions $\left(C_{t}\right)$.

Proposition 3 There exists $\tilde{\delta}$ such that, if $F_{K}(\infty, 1)<\delta<\tilde{\delta}$, any associated equilibrium is efficient.

Proof. (1) Let $\varepsilon \in(0,1)$ and $a \geq M / \varepsilon$. Then, there exists $\hat{\delta}$ such that, for any $\zeta \in\left(F_{K}(\infty, 1), \hat{\delta}\right]$, if $F_{K}\left(K_{t} / L_{t}, 1\right) \leq \zeta$, we have $K_{t} / L_{t} \geq a$. Since $K_{t} \leq M$, we obtain

$$
L_{t} \leq M / a \leq \varepsilon
$$

(2) Let $\varepsilon \in(0,1)$ and $b$ satisfy $F_{L}(1,1 / b) u_{i}^{\prime}(M)>v_{i}^{\prime}(1-\varepsilon)$ for any $i$. There exists $\bar{\delta}$ such that for any $\zeta \in\left(F_{K}(\infty, 1), \bar{\delta}\right]$, if $F_{K}\left(K_{t} / L_{t}, 1\right) \leq \zeta$, then $K_{t} / L_{t} \geq b$. Hence,

$$
F_{L}\left(1, L_{t} / K_{t}\right) u_{i}^{\prime}(M)>v_{i}^{\prime}(1-\varepsilon)
$$

for any $i$.

(3) Let $\varepsilon \in(0,1)$ and $\varkappa \in(0,1)$ satisfy $\varkappa \min \{\hat{\delta}, \bar{\delta}\}>F_{K}(\infty, 1)$. Define $\tilde{\delta} \equiv \varkappa \min \{\hat{\delta}, \bar{\delta}\}$. Take $\delta \in\left(F_{K}(\infty, 1), \tilde{\delta}\right)$. Inequality $F_{K}\left(\bar{K}_{t} / \bar{L}_{t}, 1\right)<\tilde{\delta} / \varkappa$ never holds. On the contrary, let it hold for some $t$. From (12), we get $\bar{L}_{t} \leq \varepsilon$ and, from (13),

$$
F_{L}\left(1, \bar{L}_{t} / \bar{K}_{t}\right) u_{i}^{\prime}(M)>v_{i}^{\prime}(1-\varepsilon)
$$

for any $i$. Recall the first-order conditions (8) in claim 15: for any $i, v_{i}^{\prime}\left(\bar{\lambda}_{i t}\right) \geq$ $u_{i}^{\prime}\left(\bar{c}_{i t}\right) \bar{\omega}_{t}$. Since $\bar{\lambda}_{i t} \geq 1-\varepsilon, \bar{c}_{i t} \leq M$ and $\bar{\omega}_{t}=F_{L}\left(1, \bar{L}_{t} / \bar{K}_{t}\right)$, we obtain for any 
$i: v_{i}^{\prime}(1-\varepsilon) \geq u_{i}^{\prime}(M) F_{L}\left(1, \bar{L}_{t} / \bar{K}_{t}\right)$, which is a contradiction to (14). Then, we have for any $t, \bar{\rho}_{t}=F_{K}\left(\bar{K}_{t} / \bar{L}_{t}, 1\right) \geq \tilde{\delta} / \varkappa$. We find $\bar{\rho}_{t}-\delta>\tilde{\delta} / \varkappa-\tilde{\delta}$,

$$
\bar{q}_{t}=\frac{1}{1-\delta+\bar{\rho}_{t}}<\frac{1}{1+(1 / \varkappa-1) \tilde{\delta}}<1
$$

for any $t$ and

$$
\bar{Q}_{T}=\prod_{t=1}^{T} \frac{1}{1-\delta+\bar{\rho}_{t}}<\left[\frac{1}{1+(1 / \varkappa-1) \tilde{\delta}}\right]^{T}
$$

Therefore, $\lim _{T \rightarrow+\infty} \bar{Q}_{T}=0$.

The depreciation rate of capital plays a crucial role. When $\delta$ is lower, the gross return on capital is higher and a productive economy turns out to be more efficient. However, Becker et al. (2011) provide an example with $\delta=1$, where the Ramsey equilibrium may be inefficient because of the lower returns on capital. Their construction assumes a two-household economy with capital depreciating completely within the period (i.e. $\delta=1$ : capital is circulating) and each agent's labor supply is perfectly inelastic. Their example's aggregate capital stock exhibits a period three cycle. Previously, Becker and Mitra (2011) showed each two-cycle equilibrium to be efficient when $\delta=1$ and each agent's labor supply is perfectly inelastic.

The strategy behind building their inefficiency example starts with the necessary conditions for a period three cycle. Inefficiency occurs when the compound (or geometric) returns to capital investment are negative over longer and longer horizons. This occurs because the aggregate capital stock exceeds the GoldenRule stock infinitely often. In fact, they show that the aggregate capital path can be of only one type: it attains a peak stock level above the Golden-Rule, followed by a lower capital stock also above the Golden-Rule, and then followed by a capital stock below the Golden-Rule. The implied shadow prices of capital satisfy the well-known Cass criterion (1972) for inefficiency. The detailed construction exhibits the various restrictions on the agent's utility functions and discount factors along with the needed properties on the one-sector production function that are consistent with this period three cycle picture. Those details also track the fluctuations in individual consumption. The key to completing the inefficiency example is demonstrating the more impatient household holds all the economy's capital infinitely often while the more patient one has no capital at those times. In this way, the so-called turnpike property fails to hold. Otherwise, when the turnpike property obtains (the most patient agent has all the capital eventually and the more impatient's stocks are eventually zero and remain so thereafter), then the resulting equilibrium is known to be efficient according to Becker and Mitra's (2011) theorems. Our Proposition 4, giving a sufficient condition for efficiency, is in the same spirit as the conditions offered in Becker and Mitra's paper. The difference is that we include a labor-leisure choice option for each agent in addition to admitting durable capital $(0<\delta<1)$. The labor-leisure choice plays a subsidiary role through the separability property in Assumption 5. The formal argument for the proposition depends on establishing a transversality condition (by Proposition 2) for capital goods prices obtains 
and that, in turn, only depends on there being at least one agent who eventually holds positive stocks for all time irrespective of that person's labor supply decision.

Proposition 4 If there are $i$ and $S$ such that, for any $t>S, \bar{c}_{i t} \geq c>0$ and $\bar{k}_{i t} \geq k>0$, then the equilibrium is efficient.

Proof. Consider such an individual. Since $u^{\prime}\left(\bar{c}_{i t}\right) \leq u^{\prime}(c)<+\infty$ for $t>$ $S$, we obtain $\sum_{t=0}^{\infty} \beta_{i}^{t} u_{i}^{\prime}\left(\bar{c}_{i t}\right)<+\infty$ and, because of point (7) in Claim 15, $\sum_{t=0}^{\infty} \bar{\mu}_{i t} \bar{p}_{t}<+\infty$. Then $\bar{\mu}_{i t} \bar{p}_{t} \rightarrow 0$. We know that

$$
\frac{\bar{\mu}_{i t+1} \bar{p}_{t+1}}{\bar{\mu}_{i t} \bar{p}_{t}}=\frac{1}{1-\delta+\bar{\rho}_{t+1}}=\bar{q}_{t+1}
$$

if $\bar{k}_{i t+1}>0$. Hence, for any $T>S$,

$$
\bar{Q}_{T}=\bar{Q}_{S} \prod_{t=S}^{T-1} \bar{q}_{t+1}=\bar{Q}_{S} \prod_{t=S}^{T-1} \frac{\bar{\mu}_{i t+1} \bar{p}_{t+1}}{\bar{\mu}_{i t} \bar{p}_{t}}=\bar{Q}_{S} \frac{\bar{\mu}_{i T} \bar{p}_{T}}{\bar{\mu}_{i S} \bar{p}_{S}}
$$

and, finally,

$$
\lim _{T \rightarrow+\infty} \bar{Q}_{T}=\frac{\bar{Q}_{S}}{\bar{\mu}_{i S} \bar{p}_{S}} \lim _{T \rightarrow+\infty}\left(\bar{\mu}_{i T} \bar{p}_{T}\right)=0
$$

In our model, as in Becker (1980), nondominant consumers have positive steady state consumption. Conversely, in Le Van et al. (2007), the consumption of impatient agents asymptotically vanishes. The difference rests on the nature of the budget constraint.

Le Van et al. (2007) allow intertemporal trades of future labor for current consumption in a loan market. In other terms, each consumer's budget constraint requires total assets to be nonnegative only asymptotically. Thus, impatient agents may borrow to consume more today and, since their total assets consist only of a discounted wage income from a certain period on, they experience zero consumption asymptotically.

Conversely, to exclude agents without capital from the loan market, we require capital assets to be nonnegative at each moment of time. For impatient consumers with no capital stocks, this implies that the wage income is consumed at each time.

In Le Van et al. (2007), the First Welfare Theorem holds and the competitive equilibrium is a Pareto optimum. In our case, the possibilities for intertemporal trade are restrained and the resulting competitive equilibrium is a constrained Pareto optimum. We observe that Malinvaud efficiency is different from Pareto optimality and that Proposition 4 gives only sufficient conditions for Malinvaud efficiency.

However, as shown in Bosi and Seegmuller (2010), there exists a unique steady state with $c_{i}>0$ for all $i=1, \ldots, m, k_{1}>0$ and $k_{i}=0$ for $i>1$, where $\beta_{1}>\beta_{2} \geq \ldots \geq \beta_{m}$. This distribution of capital still holds along a 
dynamic path in a neighborhood of the steady state, as soon as the inequality $u_{i}^{\prime}\left(\bar{w}_{t} \bar{l}_{i t}\right)>\beta_{i}\left(1-\delta+\bar{r}_{t+1} / \bar{p}_{t+1}\right) u_{i}^{\prime}\left(\bar{w}_{t+1} \bar{l}_{i t+1}\right)$ is satisfied for all $i>1$. Proposition 3 in Bosi and Seegmuller (2010) provides sufficient conditions for a local convergence to a steady state with positive consumption and capital for the dominant consumer (namely, a sufficiently large elasticity of capital-labor substitution or a sufficiently large impatient agents' average elasticity of labor supply). Otherwise, a stable cycle of period two arises around the steady state, now unstable. The cycle remains positive if the bifurcation parameter is close enough to the critical value. Under these conditions, positive thresholds for consumption and capital $(c, k>0)$ exist and Proposition 4 applies, that is the competitive equilibrium is Malinvaud efficient.

\section{Conclusion}

In this paper, we have shown the existence of an intertemporal equilibrium with market imperfections (borrowing constraints) and addressed the issues of bubbles existence and equilibrium efficiency.

Applying the fixed-point theorem of Gale-Mas-Colell, we have proved the existence of an equilibrium in a finite-horizon bounded economy. This equilibrium turns out to be also an equilibrium of any unbounded economy with the same fundamentals. Eventually, we have shown the existence of an equilibrium in an infinite-horizon economy as a limit of a sequence of truncated economies by applying a uniform convergence argument.

The paper generalizes in one respect Becker et al. (1991) by considering an elastic labor supply, and, in another respect, Bosi and Seegmuller (2010) by providing a proof of global existence. Our methodology, inspired by Florenzano (1999), is quite general and can be applied to other Ramsey models with different market imperfections.

At the end of the paper, we have raised the question of bubbles occurrence and efficiency. We have proved that, in our productive economy, bubbles are ruled out and that, under a sufficiently small capital depreciation rate, the equilibrium is efficient in the Malinvaud (1953) sense. Conversely, when $\delta=1$, the equilibrium may be inefficient.

\section{Appendix 1: existence of equilibrium in a finite- horizon economy}

Let us prove Proposition 1.

Define a bounded price set:

$$
P \equiv\left\{(\mathbf{p}, \mathbf{r}, \mathbf{w}):-1 \leq p_{t} \leq 1,0 \leq r_{t} \leq 1,0 \leq w_{t} \leq 1, t=0, \ldots, T\right\}
$$

At this stage, put no restriction on the sign of $p_{t}$. We will prove later price positivity through an equilibrium argument. 
Focus now on the budget constraints: $p_{t}\left[c_{i t}+k_{i t+1}-(1-\delta) k_{i t}\right] \leq r_{t} k_{i t}+$ $w_{t}\left(1-\lambda_{i t}\right)$ for $t=0, \ldots, T-1$ and $p_{T}\left[c_{i T}-(1-\delta) k_{i T}\right] \leq r_{T} k_{i T}+w_{T}\left(1-\lambda_{i T}\right)$.

In the spirit of Bergstrom (1976), we introduce the modified budget sets:

$$
\begin{gathered}
B_{i}(\mathbf{p}, \mathbf{r}, \mathbf{w}) \\
\equiv\left\{\begin{array}{c}
\left(\mathbf{c}_{i}, \mathbf{k}_{i}, \boldsymbol{\lambda}_{i}\right) \in X_{i} \times Y_{i} \times Z_{i}: \\
p_{t}\left[c_{i t}+k_{i t+1}-(1-\delta) k_{i t}\right]<r_{t} k_{i t}+w_{t}\left(1-\lambda_{i t}\right)+\gamma\left(p_{t}, r_{t}, w_{t}\right) \\
t=0, \ldots, T-1 \\
p_{T}\left[c_{i T}-(1-\delta) k_{i T}\right]<r_{T} k_{i T}+w_{T}\left(1-\lambda_{i T}\right)+\gamma\left(p_{T}, r_{T}, w_{T}\right)
\end{array}\right\} \\
\equiv\left\{\begin{array}{c}
\left(\mathbf{c}_{i}, \mathbf{k}_{i}, \boldsymbol{\lambda}_{i}\right) \in X_{i} \times Y_{i} \times Z_{i}: \\
C_{i}(\mathbf{p}, \mathbf{r}, \mathbf{w}) \\
p_{t}\left[c_{i t}+k_{i t+1}-(1-\delta) k_{i t}\right] \leq r_{t} k_{i t}+w_{t}\left(1-\lambda_{i t}\right)+\gamma\left(p_{t}, r_{t}, w_{t}\right) \\
t=0, \ldots, T-1 \\
p_{T}\left[c_{i T}-(1-\delta) k_{i T}\right] \leq r_{T} k_{i T}+w_{T}\left(1-\lambda_{i T}\right)+\gamma\left(p_{T}, r_{T}, w_{T}\right)
\end{array}\right\}
\end{gathered}
$$

where $\gamma\left(p_{t}, r_{t}, w_{t}\right) \equiv 1-\min \left\{1,\left|p_{t}\right|+r_{t}+w_{t}\right\}$. We denote by $\bar{B}_{i}(\mathbf{p}, \mathbf{r}, \mathbf{w})$ the closure of $B_{i}(\mathbf{p}, \mathbf{r}, \mathbf{w})$.

Claim 8 For every $(\mathbf{p}, \mathbf{r}, \mathbf{w}) \in P$, we have $B_{i}(\mathbf{p}, \mathbf{r}, \mathbf{w}) \neq \varnothing$ and $C_{i}(\mathbf{p}, \mathbf{r}, \mathbf{w})=$ $\bar{B}_{i}(\mathbf{p}, \mathbf{r}, \mathbf{w})$.

Proof. Without loss of generality, focus on the modified budget constraints of the first two periods:

$$
\begin{aligned}
& p_{0}\left[c_{i 0}+k_{i 1}-(1-\delta) k_{i 0}\right]<r_{0} k_{i 0}+w_{0}\left(1-\lambda_{i 0}\right)+\gamma\left(p_{0}, r_{0}, w_{0}\right) \\
& p_{1}\left[c_{i 1}+k_{i 2}-(1-\delta) k_{i 1}\right]<r_{1} k_{i 1}+w_{1}\left(1-\lambda_{i 1}\right)+\gamma\left(p_{1}, r_{1}, w_{1}\right)
\end{aligned}
$$

We know that $-1 \leq p_{t} \leq 1,0 \leq r_{t} \leq 1,0 \leq w_{t} \leq 1$.

(1) Let $\left|p_{0}\right|+r_{0}+w_{0}<1$. Then $\gamma\left(p_{0}, r_{0}, w_{0}\right)>0$.

Assume $B_{c}$ to be large enough to choose $c_{i 0}=(1-\delta) k_{i 0}$ and set $\lambda_{i 0}=1$ to remain in $X_{i} \times Z_{i}$. Inequality (15) becomes $p_{0} k_{i 1}<r_{0} k_{i 0}+\gamma\left(p_{0}, r_{0}, w_{0}\right)$ and is satisfied if $k_{i 1}>0$ is sufficiently close to zero.

Focus now on the second period and two subcases.

(1.1) Let $\left|p_{1}\right|+r_{1}+w_{1}<1$. Then $\gamma\left(p_{1}, r_{1}, w_{1}\right)>0$.

If $p_{1}<0$, choose $c_{i 1}$ sufficiently large (assume the upper bound $B_{c}$ to be large enough) and inequality (16) holds.

If $p_{1} \geq 0$, set $c_{i 1}=k_{i 2}=0$ : inequality (16) becomes $-p_{1}(1-\delta) k_{i 1}<$ $r_{1} k_{i 1}+w_{1}\left(1-\lambda_{i 1}\right)+\gamma\left(p_{1}, r_{1}, w_{1}\right)$ and is satisfied. Notice that, in this case, inequality (16) holds also if $k_{i 2}>0$ but sufficiently close to zero.

(1.2) Let $\left|p_{1}\right|+r_{1}+w_{1} \geq 1$. Then $\gamma\left(p_{1}, r_{1}, w_{1}\right)=0$.

If $p_{1}<0$, choose $c_{i 1}$ sufficiently large (assume the upper bound $B_{c}$ to be large enough) and inequality (16) is verified.

If $p_{1}=0$, choose $\lambda_{i 1}=0$. Inequality (16) becomes $0<r_{1} k_{i 1}+w_{1}$ and, since either $r_{1}>0$ or $w_{1}>0$, it is satisfied because $k_{i 1}>0$ (see point (1)).

If $p_{1}>0$, set $c_{i 1}=k_{i 2}=0$ : inequality (16) becomes $-p_{1}(1-\delta) k_{i 1}<$ $r_{1} k_{i 1}+w_{1}\left(1-\lambda_{i 1}\right)$ and is satisfied because $k_{i 1}>0$ (see point (1)) and $\delta<1$. 
Notice that, in this case, inequality (16) holds also if $k_{i 2}>0$ but sufficiently close to zero.

(2) Let $\left|p_{0}\right|+r_{0}+w_{0} \geq 1$. Then $\gamma\left(p_{0}, r_{0}, w_{0}\right)=0$.

If $p_{0}<0$, assume $B_{c}$ to be large enough to set $c_{i 0}=(1-\delta) k_{i 0}$ and choose $k_{i 1}>0$. Inequality (15) becomes $p_{0} k_{i 1}<r_{0} k_{i 0}+w_{0}\left(1-\lambda_{i 0}\right)$ and is verified.

If $p_{0}=0$, we have either $r_{0}>0$ or $w_{0}>0$. Choose $\lambda_{i 0}=0<k_{i 1}$. Inequality (15) becomes $0<r_{0} k_{i 0}+w_{0}$. We cannot exclude the case $r_{0}=0$ or $w_{0}=0$, but Assumption 3 ensures that inequality (15) holds.

If $p_{0}>0$, choose $c_{i 0}=0$ and $0<k_{i 1}<(1-\delta) k_{i 0}$. Inequality (15) becomes $p_{0}\left[k_{i 1}-(1-\delta) k_{i 0}\right]<r_{0} k_{i 0}+w_{0}\left(1-\lambda_{i 0}\right)$ and is satisfied.

Focus on the second period and two subcases.

(2.1) Let $\left|p_{1}\right|+r_{1}+w_{1}<1$. Then $\gamma\left(p_{1}, r_{1}, w_{1}\right)>0$.

Apply the same arguments of point (1.1).

(2.2) Let now $\left|p_{1}\right|+r_{1}+w_{1} \geq 1$. Then $\gamma\left(p_{1}, r_{1}, w_{1}\right)=0$.

Apply the same arguments of point (1.2) (just replace "see point (1)" with "see point (2)").

Summing up, whatever the price system $(\mathbf{p}, \mathbf{r}, \mathbf{w}) \in P$, we find a feasible allocation in the modified budget set: $\left(\mathbf{c}_{i}, \mathbf{k}_{i}, \boldsymbol{\lambda}_{i}\right) \in B_{i}(\mathbf{p}, \mathbf{r}, \mathbf{w})$. In addition, $B_{i}(\mathbf{p}, \mathbf{r}, \mathbf{w}) \neq \varnothing$ implies $C_{i}(\mathbf{p}, \mathbf{r}, \mathbf{w})=\bar{B}_{i}(\mathbf{p}, \mathbf{r}, \mathbf{w})$ for every $(\mathbf{p}, \mathbf{r}, \mathbf{w}) \in P$.

Claim $9 B_{i}$ is a lower semi-continuous correspondence on $P$.

Proof. Simply observe that $B_{i}$ has an open graph.

Claim $10 C_{i}$ is upper semi-continuous on $P$ with compact convex values.

Proof. The inequalities that appear in the definition of $C_{i}$, are affine and $X_{i}^{T} \times Y_{i}^{T} \times Z_{i}^{T}$ is a compact convex set. Hence, $C_{i}$ has a compact graph with convex values in a fixed compact set and turns out to be upper semicontinuous.

In the spirit of Gale and Mas-Colell $(1975,1979)$, we introduce the reaction correspondences $\varphi_{i}\left(\mathbf{p}, \mathbf{r}, \mathbf{w},\left(\mathbf{c}_{h}, \mathbf{k}_{h}, \boldsymbol{\lambda}_{h}\right)_{h=1}^{m}, \mathbf{K}, \mathbf{L}\right), i=0, \ldots, m+1$ defined on $P \times\left[\times_{h=1}^{m}\left(X_{h} \times Y_{h} \times Z_{h}\right)\right] \times Y \times Z$, where $i=0$ denotes an additional agent, the auctioneer, $i=1, \ldots, m$ the consumers, and $i=m+1$ the firm. These correspondences are defined as follows.

Agent $i=0$ (the auctioneer):

$$
\begin{aligned}
& \varphi_{0}\left(\mathbf{p}, \mathbf{r}, \mathbf{w},\left(\mathbf{c}_{h}, \mathbf{k}_{h}, \boldsymbol{\lambda}_{h}\right)_{h=1}^{m}, \mathbf{K}, \mathbf{L}\right) \\
& \equiv\left\{\begin{array}{c}
(\tilde{\mathbf{p}}, \tilde{\mathbf{r}}, \tilde{\mathbf{w}}) \in P: \\
\sum_{t=0}^{T}\left(\tilde{p}_{t}-p_{t}\right)\left(\sum_{i=1}^{m}\left[c_{i t}+k_{i t+1}-(1-\delta) k_{i t}\right]-F\left(K_{t}, L_{t}\right)\right) \\
+\sum_{t=0}^{T}\left(\tilde{r}_{t}-r_{t}\right)\left(K_{t}-\sum_{i=1}^{m} k_{i t}\right) \\
+\sum_{t=0}^{T}\left(\tilde{w}_{t}-w_{t}\right)\left(L_{t}-m+\sum_{i=1}^{m} \lambda_{i t}\right)>0
\end{array}\right\}(17)
\end{aligned}
$$

Agents $i=1, \ldots, m$ (consumers-workers):

$$
\begin{aligned}
& \varphi_{i}\left(\mathbf{p}, \mathbf{r}, \mathbf{w},\left(\mathbf{c}_{h}, \mathbf{k}_{h}, \boldsymbol{\lambda}_{h}\right)_{h=1}^{m}, \mathbf{K}, \mathbf{L}\right) \\
\equiv & \left\{\begin{array}{c}
B_{i}(\mathbf{p}, \mathbf{r}, \mathbf{w}) \text { if }\left(\mathbf{c}_{i}, \mathbf{k}_{i}, \boldsymbol{\lambda}_{i}\right) \notin C_{i}(\mathbf{p}, \mathbf{r}, \mathbf{w}) \\
B_{i}(\mathbf{p}, \mathbf{r}, \mathbf{w}) \cap\left[P_{i}\left(\mathbf{c}_{i}, \boldsymbol{\lambda}_{i}\right) \times Y_{i}\right] \text { if }\left(\mathbf{c}_{i}, \mathbf{k}_{i}, \boldsymbol{\lambda}_{i}\right) \in C_{i}(\mathbf{p}, \mathbf{r}, \mathbf{w})
\end{array}\right\}
\end{aligned}
$$


where $P_{i}$ is the $i$ th agent's set of strictly preferred allocations: $P_{i}\left(\mathbf{c}_{i}, \boldsymbol{\lambda}_{i}\right) \equiv$ $\left\{\left(\tilde{\mathbf{c}}_{i}, \tilde{\boldsymbol{\lambda}}_{i}\right): \sum_{t=0}^{T} \beta_{i}^{t} u_{i}\left(\tilde{c}_{i t}, \tilde{\lambda}_{i t}\right)>\sum_{t=0}^{T} \beta_{i}^{t} u_{i}\left(c_{i t}, \lambda_{i t}\right)\right\}$.

Agent $i=m+1$ (the firm):

$$
\begin{gathered}
\varphi_{m+1}\left(\mathbf{p}, \mathbf{r}, \mathbf{w},\left(\mathbf{c}_{h}, \mathbf{k}_{h}, \boldsymbol{\lambda}_{h}\right)_{h=1}^{m}, \mathbf{K}, \mathbf{L}\right) \\
\equiv\left\{\begin{array}{c}
(\tilde{\mathbf{K}}, \tilde{\mathbf{L}}) \in Y \times Z: \\
\sum_{t=0}^{T}\left[p_{t} F\left(\tilde{K}_{t}, \tilde{L}_{t}\right)-r_{t} \tilde{K}_{t}-w_{t} \tilde{L}_{t}\right] \\
>\sum_{t=0}^{T}\left[p_{t} F\left(K_{t}, L_{t}\right)-r_{t} K_{t}-w_{t} L_{t}\right]
\end{array}\right\}
\end{gathered}
$$

We observe that $\varphi_{i}: \Phi \rightarrow 2^{\Phi_{i}}$ where

$$
\begin{aligned}
\Phi & \equiv \Phi_{0} \times \ldots \times \Phi_{m+1} \\
\Phi_{0} & \equiv P \\
\Phi_{i} & \equiv X_{i} \times Y_{i} \times Z_{i}, i=1, \ldots, m \\
\Phi_{m+1} & \equiv Y \times Z
\end{aligned}
$$

and $2^{\Phi_{i}}$ denotes the set of subsets of $\Phi_{i}$.

Claim $11 \varphi_{i}$ is a lower semi-continuous convex-valued correspondence for $i=$ $0, \ldots, m+1$.

\section{Proof.}

(1) Focus first on openness.

$\varphi_{0}$ has an open graph.

Consider $\varphi_{i}$ with $i=1, \ldots, m . B_{i}$ is lower semi-continuous and has an open graph in $X_{i} \times Y_{i} \times Z_{i}$ (Claim 9). $P_{i}\left(\mathbf{c}_{i}, \boldsymbol{\lambda}_{i}\right)$ has also an open graph in $X_{i} \times Z_{i}$, so $B_{i}(\mathbf{p}, \mathbf{r}, \mathbf{w}) \cap\left[P_{i}\left(\mathbf{c}_{i}, \boldsymbol{\lambda}_{i}\right) \times Y_{i}\right]$ has an open graph in $X_{i} \times Y_{i} \times Z_{i}$.

$\varphi_{m+1}$ has an open graph.

(2) Focus now on convexity.

The affinity of the function with respect to $(\tilde{\mathbf{p}}, \tilde{\mathbf{r}}, \tilde{\mathbf{w}})$ in the left-hand side of the inequality defining $\varphi_{0}$ implies the convexity of $\varphi_{0}$.

The affinity of the modified budget constraint implies the convexity of $B_{i}$ for every $(\mathbf{p}, \mathbf{r}, \mathbf{w}) \in P$. The concavity of $u_{i}$ implies the convexity of $P_{i}\left(\mathbf{c}_{i}, \boldsymbol{\lambda}_{i}\right)$ for every $\left(\mathbf{c}_{i}, \boldsymbol{\lambda}_{i}\right) \in X_{i} \times Z_{i}$. Then $B_{i}(\mathbf{p}, \mathbf{r}, \mathbf{w}) \cap\left[P_{i}\left(\mathbf{c}_{i}, \boldsymbol{\lambda}_{i}\right) \times Y_{i}\right]$ is convex and $\varphi_{i}$ is convex-valued for $i=1, \ldots, m$.

Concavity of $F$ implies also the convexity of $\varphi_{m+1}$.

Let us simplify the notation

$$
\begin{aligned}
\mathbf{v} & \equiv\left(\mathbf{p}, \mathbf{r}, \mathbf{w},\left(\mathbf{c}_{h}, \mathbf{k}_{h}, \boldsymbol{\lambda}_{h}\right)_{h=1}^{m}, \mathbf{K}, \mathbf{L}\right) \\
\mathbf{v}_{0} & \equiv(\mathbf{p}, \mathbf{r}, \mathbf{w}) \\
\mathbf{v}_{i} & \equiv\left(\mathbf{c}_{i}, \mathbf{k}_{i}, \boldsymbol{\lambda}_{i}\right) \text { for } i=1, \ldots, m \\
\mathbf{v}_{m+1} & \equiv(\mathbf{K}, \mathbf{L})
\end{aligned}
$$

Definition and properties of correspondence $\varphi$ satisfy the assumptions of the Gale and Mas-Colell (1975) fixed-point theorem and allow us to prove the main result of the paper, that is the existence of a general equilibrium. 
Lemma 3 (fixed-point argument) There exists $\mathbf{v} \in \Phi$ such that either $\varphi_{i}(\mathbf{v})=$ $\varnothing$ or $\mathbf{v}_{i} \in \varphi_{i}(\mathbf{v})$ for $i=0, \ldots, m+1$.

Proof. $\Phi$ is a non-empty compact convex subset of $\mathbb{R}^{m T+(5+2 m)(T+1)}$. Each $\varphi_{i}: \Phi \rightarrow 2^{\Phi_{i}}$ is a convex (possibly empty) valued correspondence whose graph is open in $\Phi \times \Phi_{i}$ (Claim 11). Then the Gale and Mas-Colell (1975) fixed-point theorem applies.

We observe the following.

(1) By definition of $\varphi_{0}$ (the inequality in (17) is strict): (p, r, w) $\notin \varphi_{0}(\mathbf{v})$.

(2) $\left(\mathbf{c}_{i}, \mathbf{k}_{i}, \boldsymbol{\lambda}_{i}\right) \notin P_{i}\left(\mathbf{c}_{i}, \boldsymbol{\lambda}_{i}\right) \times Y_{i}$ implies that $\left(\mathbf{c}_{i}, \mathbf{k}_{i}, \boldsymbol{\lambda}_{i}\right) \notin \varphi_{i}(\mathbf{v})$ for $i=$ $1, \ldots, m$.

(3) By definition of $\varphi_{m+1}$ (the inequality in (18) is also strict): $(\mathbf{K}, \mathbf{L}) \notin$ $\varphi_{m+1}(\mathbf{v})$.

Then, for $i=0, \ldots, m+1, \mathbf{v}_{i} \notin \varphi_{i}(\mathbf{v})$.

According to Lemma 3 , there exists $\overline{\mathbf{v}} \in \Phi$ such that $\varphi_{i}(\overline{\mathbf{v}})=\varnothing$ for $i=$ $0, \ldots, m+1$, that is, there exists $\overline{\mathbf{v}} \in \Phi$ such that the following holds.

Focus on agent $i=0$. For every $(\mathbf{p}, \mathbf{r}, \mathbf{w}) \in P$,

$$
\begin{aligned}
& \sum_{t=0}^{T}\left(p_{t}-\bar{p}_{t}\right)\left(\sum_{i=1}^{m}\left[\bar{c}_{i t}+\bar{k}_{i t+1}-(1-\delta) \bar{k}_{i t}\right]-F\left(\bar{K}_{t}, \bar{L}_{t}\right)\right) \\
& +\sum_{t=0}^{T}\left(r_{t}-\bar{r}_{t}\right)\left(\bar{K}_{t}-\sum_{i=1}^{m} \bar{k}_{i t}\right)+\sum_{t=0}^{T}\left(w_{t}-\bar{w}_{t}\right)\left(\bar{L}_{t}-m+\sum_{i=1}^{m} \bar{\lambda}_{i t}\right) \\
\leq & 0
\end{aligned}
$$

Consider consumers $i=1, \ldots, m .\left(\overline{\mathbf{c}}_{i}, \overline{\mathbf{k}}_{i}, \overline{\boldsymbol{\lambda}}_{i}\right) \in C_{i}(\overline{\mathbf{p}}, \overline{\mathbf{r}}, \overline{\mathbf{w}})$ and $B_{i}(\overline{\mathbf{p}}, \overline{\mathbf{r}}, \overline{\mathbf{w}}) \cap$ $\left[P_{i}\left(\overline{\mathbf{c}}_{i}, \overline{\boldsymbol{\lambda}}_{i}\right) \times Y_{i}\right]=\varnothing$ for $i=1, \ldots, m$. Then, for $i=1, \ldots, m,\left(\mathbf{c}_{i}, \mathbf{k}_{i}, \boldsymbol{\lambda}_{i}\right) \in$ $C_{i}(\overline{\mathbf{p}}, \overline{\mathbf{r}}, \overline{\mathbf{w}})=\bar{B}_{i}(\overline{\mathbf{p}}, \overline{\mathbf{r}}, \overline{\mathbf{w}})$ implies

$$
\sum_{t=0}^{T} \beta_{i}^{t} u_{i}\left(c_{i t}, \lambda_{i t}\right) \leq \sum_{t=0}^{T} \beta_{i}^{t} u_{i}\left(\bar{c}_{i t}, \bar{\lambda}_{i t}\right)
$$

Focus on the firm $i=m+1$. For $t=0, \ldots, T$ and for every $(\mathbf{K}, \mathbf{L}) \in Y \times Z$, we have $\sum_{t=0}^{T}\left[\bar{p}_{t} F\left(K_{t}, L_{t}\right)-\bar{r}_{t} K_{t}-\bar{w}_{t} L_{t}\right] \leq \sum_{t=0}^{T}\left[\bar{p}_{t} F\left(\bar{K}_{t}, \bar{L}_{t}\right)-\bar{r}_{t} \bar{K}_{t}-\bar{w}_{t} \bar{L}_{t}\right]$.

This is possible if and only if

$$
\bar{p}_{t} F\left(K_{t}, L_{t}\right)-\bar{r}_{t} K_{t}-\bar{w}_{t} L_{t} \leq \bar{p}_{t} F\left(\bar{K}_{t}, \bar{L}_{t}\right)-\bar{r}_{t} \bar{K}_{t}-\bar{w}_{t} \bar{L}_{t}
$$

for any $t$ (simply choose $(\mathbf{K}, \mathbf{L})$ such that $\left(K_{s}, L_{s}\right)=\left(\bar{K}_{s}, \bar{L}_{s}\right)$ if $s \neq t$, to prove the necessity, and sum (21) side by side to prove the sufficiency). In particular, the equilibrium profit is nonnegative.

$$
\bar{p}_{t} F\left(\bar{K}_{t}, \bar{L}_{t}\right)-\bar{r}_{t} \bar{K}_{t}-\bar{w}_{t} \bar{L}_{t} \geq 0
$$

In the following claim, we prove that input excess demands are nonnegative too. 
Claim 12 If $\bar{p}_{t}>0$, then $\bar{K}_{t}-\sum_{i=1}^{m} \bar{k}_{i t} \geq 0$ and $\bar{L}_{t}-\sum_{i=1}^{m} \bar{l}_{i t} \geq 0$.

Proof.

(1) We notice that, from (19), if the demand for capital is less than the supply of capital: $\bar{K}_{t}<\sum_{i=1}^{m} \bar{k}_{i t}$, we get $\bar{r}_{t}=0$. But, since $\bar{p}_{t}>0, \bar{r}_{t}=0$ implies $\bar{K}_{t}=B_{K}$ and, so, $B_{K}=\bar{K}_{t}<\sum_{i=1}^{m} \bar{k}_{i t} \leq A<B_{K}$, a contradiction. Then $\bar{K}_{t}-\sum_{i=1}^{m} \bar{k}_{i t} \geq 0$ for $t=0, \ldots, T+1$.

(2) Similarly, we notice that, if the labor demand is less than the labor supply: $\bar{L}_{t}<\sum_{i=1}^{m} \bar{l}_{i t}$, we have $\bar{w}_{t}=0$. But $\bar{w}_{t}=0$ implies $\bar{L}_{t}=B_{L}$ and, so, $B_{L}=\bar{L}_{t}<\sum_{i=1}^{m} \bar{l}_{i t} \leq m<B_{L}$, a contradiction. Then $\bar{L}_{t}-\sum_{i=1}^{m} \bar{l}_{i t} \geq 0$ for $t=0, \ldots, T+1$.

Let $\bar{Z}_{t} \equiv \sum_{i=1}^{m}\left[\bar{c}_{i t}+\bar{k}_{i t+1}-(1-\delta) \bar{k}_{i t}\right]-F\left(\bar{K}_{t}, \bar{L}_{t}\right)$ be the aggregate excess demand for goods at time $t$. We want to prove that $\bar{Z}_{t}=0$.

Assume, by contradiction, that

$$
\bar{Z}_{t} \neq 0
$$

Claim 13 If $\bar{Z}_{t} \neq 0$ and $p_{t} \bar{Z}_{t} \leq \bar{p}_{t} \bar{Z}_{t}$ for every $p_{t}$ with $\left|p_{t}\right| \leq 1$, then (1) $\left|\bar{p}_{t}\right|=1$ and (2) $\bar{p}_{t} \bar{Z}_{t}>0$.

\section{Proof.}

(1) Let us show that $-1<\bar{p}_{t}<1$ leads to a contradiction.

(1.1) If $\bar{Z}_{t}>0$, we choose $p_{t}$ such that $\bar{p}_{t}<p_{t}<1$ and we find $\bar{p}_{t} \bar{Z}_{t}<p_{t} \bar{Z}_{t}$, a contradiction.

(1.2) If $\bar{Z}_{t}<0$, we choose $p_{t}$ such that $-1<p_{t}<\bar{p}_{t}$ and we find $\bar{p}_{t} \bar{Z}_{t}<p_{t} \bar{Z}_{t}$, a contradiction.

(2) Clearly, if we choose $p_{t}=0$, we have always $\bar{p}_{t} \bar{Z}_{t} \geq 0$. Since $\bar{p}_{t}= \pm 1$ and $\bar{Z}_{t} \neq 0$, then $\bar{p}_{t} \bar{Z}_{t} \neq 0$ and, so, $\bar{p}_{t} \bar{Z}_{t}>0$.

Claim 14 If $\bar{Z}_{t} \neq 0$, then $\bar{Z}_{t}>0$ and, hence, $\bar{p}_{t}=1$.

Proof. First, we observe that (19) holds also with $p_{t}=\bar{p}_{t}$ for $t \neq s$ and $\left(r_{t}, w_{t}\right)=\left(\bar{r}_{t}, \bar{w}_{t}\right)$ for $t=0, \ldots, T$, that is

$$
\left(p_{s}-\bar{p}_{s}\right)\left(\sum_{i=1}^{m}\left[\bar{c}_{i s}+\bar{k}_{i s+1}-(1-\delta) \bar{k}_{i s}\right]-F\left(\bar{K}_{s}, \bar{L}_{s}\right)\right)=\left(p_{s}-\bar{p}_{s}\right) \bar{Z}_{s} \leq 0
$$

for every $p_{s}$ with $\left|p_{s}\right| \leq 1$. Replacing $s$ by $t$, we have $p_{t} \bar{Z}_{t} \leq \bar{p}_{t} \bar{Z}_{t}$ for every $p_{t}$ with $\left|p_{t}\right| \leq 1$.

Claim 13 applies. Then $\left|\bar{p}_{t}\right|=1$ and $\bar{p}_{t} \bar{Z}_{t}>0$.

Suppose that the conclusion of Claim 14 is false, that is $\bar{Z}_{t}<0$ and, hence, $\bar{p}_{t}=-1$. We obtain $\sum_{i=1}^{m}\left[\bar{c}_{i t}+\bar{k}_{i t+1}-(1-\delta) \bar{k}_{i t}\right]-F\left(\bar{K}_{t}, \bar{L}_{t}\right)<0$.

But if $\bar{p}_{t}=-1$, we have $\bar{c}_{i t}=B_{c}$. Indeed, if $\bar{c}_{i t}<B_{c}$ for at least one agent, we can find $\bar{c}_{i t}<c_{i t}<B_{c}$ such that $\sum_{t=0}^{T} \beta_{i}^{t} u_{i}\left(c_{i t}, \lambda_{i t}\right)>\sum_{t=0}^{T} \beta_{i}^{t} u_{i}\left(\bar{c}_{i t}, \bar{\lambda}_{i t}\right)$ 
with $\left(\mathbf{c}_{i}, \mathbf{k}_{i}, \boldsymbol{\lambda}_{i}\right) \in B_{i}(\overline{\mathbf{p}}, \overline{\mathbf{r}}, \overline{\mathbf{w}})$, against the definition of $\overline{\mathbf{v}}$ (see (20)). Then

$$
\begin{aligned}
m B_{c} & =\sum_{i=1}^{m} \bar{c}_{i t}<F\left(\bar{K}_{t}, \bar{L}_{t}\right)+(1-\delta) \sum_{i=1}^{m} \bar{k}_{i t}-\sum_{i=1}^{m} \bar{k}_{i t+1} \\
& \leq F\left(\sum_{i=1}^{m} \bar{k}_{i t}, \sum_{i=1}^{m} \bar{l}_{i t}\right)+(1-\delta) \sum_{i=1}^{m} \bar{k}_{i t} \leq F(A, m)+(1-\delta) A \leq A
\end{aligned}
$$

a contradiction.

Let us show eventually that good and input markets clear through Propositions 5 and 7 .

Proposition 5 The goods market clears: $\bar{Z}_{t}=0$, that is

$$
\sum_{i=1}^{m}\left[\bar{c}_{i t}+\bar{k}_{i t+1}-(1-\delta) \bar{k}_{i t}\right]=F\left(\bar{K}_{t}, \bar{L}_{t}\right)
$$

Proof. $\bar{p}_{t}=1$ implies $\gamma\left(\bar{p}_{t}, \bar{r}_{t}, \bar{w}_{t}\right)=0$. In this case, $\left(\overline{\mathbf{c}}_{i}, \overline{\mathbf{k}}_{i}, \overline{\boldsymbol{\lambda}}_{i}\right) \in C_{i}(\overline{\mathbf{p}}, \overline{\mathbf{r}}, \overline{\mathbf{w}})$ entails $\bar{p}_{t}\left[\bar{c}_{i t}+\bar{k}_{i t+1}-(1-\delta) \bar{k}_{i t}\right] \leq \bar{r}_{t} \bar{k}_{i t}+\bar{w}_{t}\left(1-\bar{\lambda}_{i t}\right)$ and, therefore, we have

$$
\bar{p}_{t} \sum_{i=1}^{m}\left[\bar{c}_{i t}+\bar{k}_{i t+1}-(1-\delta) \bar{k}_{i t}\right] \leq \bar{r}_{t} \sum_{i=1}^{m} \bar{k}_{i t}+\bar{w}_{t} \sum_{i=1}^{m} \bar{l}_{i t}
$$

Assume, by contradiction, $\bar{Z}_{t} \neq 0$. Claim 14 implies $\bar{p}_{t}=1$ and $\bar{Z}_{t}>0$. This implies, in turn, $\bar{p}_{t} \sum_{i=1}^{m}\left[\bar{c}_{i t}+\bar{k}_{i t+1}-(1-\delta) \bar{k}_{i t}\right]>\bar{p}_{t} F\left(\bar{K}_{t}, \bar{L}_{t}\right)$.

According to (22), we have also $\bar{p}_{t} F\left(\bar{K}_{t}, \bar{L}_{t}\right) \geq \bar{r}_{t} \bar{K}_{t}+\bar{w}_{t} \bar{L}_{t}$.

Finally, we know that $\bar{K}_{t} \geq \sum_{i=1}^{m} \bar{k}_{i t}$ and $\bar{L}_{t} \geq \sum_{i=1}^{m} \bar{l}_{i t}$ (Claim 12).

Putting together, we have $\bar{p}_{t} \sum_{i=1}^{m}\left[\bar{c}_{i t}+\bar{k}_{i t+1}-(1-\delta) \bar{k}_{i t}\right]>\bar{r}_{t} \sum_{i=1}^{m} \bar{k}_{i t}+$ $\bar{w}_{t} \sum_{i=1}^{m} \bar{l}_{i t}$, in contradiction with (24). Thus inequality (23) is false and $\bar{Z}_{t}=0$.

We observe that

$$
\begin{aligned}
\sum_{i=1}^{m} \bar{c}_{i t} & =F\left(\bar{K}_{t}, \bar{L}_{t}\right)+\sum_{i=1}^{m}\left[(1-\delta) \bar{k}_{i t}-\bar{k}_{i t+1}\right] \\
& \leq F\left(\sum_{i=1}^{m} \bar{k}_{i t}, \sum_{i=1}^{m} \bar{l}_{i t}\right)+(1-\delta) \sum_{i=1}^{m} \bar{k}_{i t} \\
& \leq F(A, m)+(1-\delta) A \leq A<B_{c}
\end{aligned}
$$

We have now to prove that capital and the labor markets clear too. The proof rests on price positivity.

Proposition $6 \bar{p}_{t}>0, t=0, \ldots, T$.

Proof. Let us show that $\bar{p}_{t}>0$. Indeed, if $\bar{p}_{t} \leq 0$, then $\bar{c}_{i t}=B_{c}$ for every $i$ and $\sum_{i=1}^{m}\left(\bar{c}_{i t}+\bar{k}_{i t+1}\right) \geq B_{c}>F(A, m)+(1-\delta) A \geq F\left(\bar{K}_{t}, \bar{L}_{t}\right)+(1-\delta) \sum_{i=1}^{m} \bar{k}_{i t}$, in contradiction with $\bar{Z}_{t}=0$. 
Proposition $7 \bar{K}_{t}=\sum_{i=1}^{m} \bar{k}_{i t}$ and $\bar{L}_{t}=\sum_{i=1}^{m} \bar{l}_{i t}$.

Proof. Since $\bar{p}_{t}>0$, we have $\bar{K}_{t} \geq \sum_{i=1}^{m} \bar{k}_{i t}$ (Claim 12). If $\bar{K}_{t}>\sum_{i=1}^{m} \bar{k}_{i t}$, from (19), we get $\bar{r}_{t}=1>0$. Then

$$
\begin{aligned}
\bar{p}_{t} \sum_{i=1}^{m}\left[\bar{c}_{i t}+\bar{k}_{i t+1}-(1-\delta) \bar{k}_{i t}\right] & =\bar{p}_{t} F\left(\bar{K}_{t}, \bar{L}_{t}\right) \geq \bar{r}_{t} \bar{K}_{t}+\bar{w}_{t} \bar{L}_{t} \\
& >\bar{r}_{t} \sum_{i=1}^{m} \bar{k}_{i t}+\bar{w}_{t} \sum_{i=1}^{m} \bar{l}_{i t}
\end{aligned}
$$

But $\left(\overline{\mathbf{c}}_{i}, \overline{\mathbf{k}}_{i}, \overline{\boldsymbol{\lambda}}_{i}\right) \in C_{i}(\overline{\mathbf{p}}, \overline{\mathbf{r}}, \overline{\mathbf{w}})$ implies $\bar{p}_{t} \sum_{i=1}^{m}\left[\bar{c}_{i t}+\bar{k}_{i t+1}-(1-\delta) \bar{k}_{i t}\right] \leq$ $\bar{r}_{t} \sum_{i=1}^{m} \bar{k}_{i t}+\bar{w}_{t} \sum_{i=1}^{m}\left(1-\bar{\lambda}_{i t}\right)$, a contradiction. Then $\bar{K}_{t}=\sum_{i=1}^{m} \bar{k}_{i t}$.

We know that $\bar{L}_{t} \geq \sum_{i=1}^{m} \bar{l}_{i t}$ (Claim 12). If $\bar{L}_{t}>\sum_{i=1}^{m} \bar{l}_{i t}$, we get $\bar{w}_{t}=1>0$. Then

$$
\begin{aligned}
\bar{p}_{t} \sum_{i=1}^{m}\left[\bar{c}_{i t}+\bar{k}_{i t+1}-(1-\delta) \bar{k}_{i t}\right] & =\bar{p}_{t} F\left(\bar{K}_{t}, \bar{L}_{t}\right) \geq \bar{r}_{t} \bar{K}_{t}+\bar{w}_{t} \bar{L}_{t} \\
& >\bar{r}_{t} \sum_{i=1}^{m} \bar{k}_{i t}+\bar{w}_{t} \sum_{i=1}^{m} \bar{l}_{i t}
\end{aligned}
$$

But $\left(\overline{\mathbf{c}}_{i}, \overline{\mathbf{k}}_{i}, \overline{\boldsymbol{\lambda}}_{i}\right) \in C_{i}(\overline{\mathbf{p}}, \overline{\mathbf{r}}, \overline{\mathbf{w}})$ implies $\bar{p}_{t} \sum_{i=1}^{m}\left[\bar{c}_{i t}+\bar{k}_{i t+1}-(1-\delta) \bar{k}_{i t}\right] \leq$ $\bar{r}_{t} \sum_{i=1}^{m} \bar{k}_{i t}+\bar{w}_{t} \sum_{i=1}^{m}\left(1-\bar{\lambda}_{i t}\right)$, a contradiction. Then $\bar{L}_{t}=\sum_{i=1}^{m} \bar{l}_{i t}$.

To prove the zero-profit condition and input prices positivity, let us observe that $\sum_{i=1}^{m} \bar{k}_{i t} \leq A<B_{K}$ and $\sum_{i=1}^{m} \bar{l}_{i t} \leq m<B_{L}$.

Proposition 8 At the prices $\left(\bar{p}_{t}, \bar{r}_{t}, \bar{w}_{t}\right)$, the equilibrium demand $\left(\bar{K}_{t}, \bar{L}_{t}\right)$ satisfies the zero-profit condition:

$$
\bar{p}_{t} F\left(\bar{K}_{t}, \bar{L}_{t}\right)=\bar{r}_{t} \bar{K}_{t}+\bar{w}_{t} \bar{L}_{t}
$$

Proof. From (22), we know that $\bar{p}_{t} F\left(\bar{K}_{t}, \bar{L}_{t}\right)-\bar{r}_{t} \bar{K}_{t}-\bar{w}_{t} \bar{L}_{t} \geq 0$. Suppose, by contradiction, that $\bar{p}_{t} F\left(\bar{K}_{t}, \bar{L}_{t}\right)-\bar{r}_{t} \bar{K}_{t}-\bar{w}_{t} \bar{L}_{t}>0$. Choose a new vector of inputs $\left(\mu \bar{K}_{t}, \mu \bar{L}_{t}\right)$ with $\mu>1$ (this is possible since $\bar{K}_{t}<B_{K}$ and $\bar{L}_{t}<B_{L}$ ). The constant returns to scale imply

$$
\begin{aligned}
\bar{p}_{t} F\left(\mu \bar{K}_{t}, \mu \bar{L}_{t}\right)-\bar{r}_{t} \mu \bar{K}_{t}-\bar{w}_{t} \mu \bar{L}_{t} & =\mu\left[\bar{p}_{t} F\left(\bar{K}_{t}, \bar{L}_{t}\right)-\bar{r}_{t} \bar{K}_{t}-\bar{w}_{t} \bar{L}_{t}\right] \\
& >\bar{p}_{t} F\left(\bar{K}_{t}, \bar{L}_{t}\right)-\bar{r}_{t} \bar{K}_{t}-\bar{w}_{t} \bar{L}_{t}
\end{aligned}
$$

against the fact that inequality $(21)$ holds for every $\left(K_{t}, L_{t}\right) \in\left[0, B_{K}\right] \times\left[0, B_{L}\right]$.

Proposition $9 \bar{r}_{t}>0, \bar{w}_{t}>0, t=0, \ldots, T$.

Proof. Recall that

$$
\bar{p}_{t} F\left(\bar{K}_{t}, \bar{L}_{t}\right)-\bar{r}_{t} \bar{K}_{t}-\bar{w}_{t} \bar{L}_{t} \geq \bar{p}_{t} F\left(K_{t}, L_{t}\right)-\bar{r}_{t} K_{t}-\bar{w}_{t} L_{t}
$$


for any pair $\left(K_{t}, L_{t}\right) \in\left[0, B_{K}\right] \times\left[0, B_{L}\right]($ see $(21))$.

Consider now $\left(K_{t}, L_{t}\right) \in \mathbb{R}_{+}^{2}$ and define $K_{t}(\gamma) \equiv \gamma K_{t}+(1-\gamma) \bar{K}_{t}$ and $L_{t}(\gamma) \equiv \gamma L_{t}+(1-\gamma) \bar{L}_{t}$ with $\gamma \in(0,1)$. Since $\bar{K}_{t}<A \leq B_{K}$ and $\bar{L}_{t}<m \leq$ $B_{L}$, we have, for $\gamma$ sufficiently close to $0,\left(K_{t}(\gamma), L_{t}(\gamma)\right) \in\left[0, B_{K}\right] \times\left[0, B_{L}\right]$ and

$$
\bar{p}_{t} F\left(\bar{K}_{t}, \bar{L}_{t}\right)-\bar{r}_{t} \bar{K}_{t}-\bar{w}_{t} \bar{L}_{t} \geq \bar{p}_{t} F\left(K_{t}(\gamma), L_{t}(\gamma)\right)-\bar{r}_{t} K_{t}(\gamma)-\bar{w}_{t} L_{t}(\gamma)
$$

The concavity of $F$ implies:

$$
\begin{aligned}
& \bar{p}_{t} F\left(K_{t}(\gamma), L_{t}(\gamma)\right)-\bar{r}_{t} K_{t}(\gamma)-\bar{w}_{t} L_{t}(\gamma) \\
\geq & \gamma\left[\bar{p}_{t} F\left(K_{t}, L_{t}\right)-\bar{r}_{t} K_{t}-\bar{w}_{t} L_{t}\right]+(1-\gamma)\left[\bar{p}_{t} F\left(\bar{K}_{t}, \bar{L}_{t}\right)-\bar{r}_{t} \bar{K}_{t}-\bar{w}_{t} \bar{L}_{t}\right]
\end{aligned}
$$

Combining with (27), we obtain

$$
\bar{p}_{t} F\left(\bar{K}_{t}, \bar{L}_{t}\right)-\bar{r}_{t} \bar{K}_{t}-\bar{w}_{t} \bar{L}_{t} \geq \bar{p}_{t} F\left(K_{t}, L_{t}\right)-\bar{r}_{t} K_{t}-\bar{w}_{t} L_{t}
$$

Assume that $\bar{w}_{t} \geq \bar{r}_{t}=0$. Fix $L_{t}>0$ and let $K_{t} \rightarrow+\infty$. The right-hand side of (28) goes to infinity, a contradiction.

A similar proof works when $\bar{r}_{t} \geq \bar{w}_{t}=0$.

We conclude Appendix 1, noticing that, at equilibrium, the artificial budget constraint à la Bergstrom (1976) we needed to apply the Gale and Mas-Colell (1975) fixed-point argument, actually collapses in the ordinary one.

Proposition 10 The modified budget constraint at equilibrium is a budget constraint: $\gamma\left(\bar{p}_{t}, \bar{r}_{t}, \bar{w}_{t}\right)=0$ for $t=0, \ldots, T$.

Proof. $\bar{p}_{t}>0$ entails that the modified budget constraint is binding:

$$
\bar{p}_{t}\left[\bar{c}_{i t}+\bar{k}_{i t+1}-(1-\delta) \bar{k}_{i t}\right]=\bar{r}_{t} \bar{k}_{i t}+\bar{w}_{t} \bar{l}_{i t}+\gamma\left(\bar{p}_{t}, \bar{r}_{t}, \bar{w}_{t}\right)
$$

This gives

$$
\bar{p}_{t} \sum_{i=1}^{m}\left[\bar{c}_{i t}+\bar{k}_{i t+1}-(1-\delta) \bar{k}_{i t}\right]=\bar{r}_{t} \sum_{i=1}^{m} \bar{k}_{i t}+\bar{w}_{t} \sum_{i=1}^{m} \bar{l}_{i t}+m \gamma\left(\bar{p}_{t}, \bar{r}_{t}, \bar{w}_{t}\right)
$$

Proposition 5 implies $\bar{p}_{t} F\left(\bar{K}_{t}, \bar{L}_{t}\right)=\bar{r}_{t} \sum_{i=1}^{m} \bar{k}_{i t}+\bar{w}_{t} \sum_{i=1}^{m} \bar{l}_{i t}+m \gamma\left(\bar{p}_{t}, \bar{r}_{t}, \bar{w}_{t}\right)$, while Propositions 8 and $7, \bar{p}_{t} F\left(\bar{K}_{t}, \bar{L}_{t}\right)=\bar{r}_{t} \sum_{i=1}^{m} \bar{k}_{i t}+\bar{w}_{t} \sum_{i=1}^{m} \bar{l}_{i t}$. Eventually, we obtain $\gamma\left(\bar{p}_{t}, \bar{r}_{t}, \bar{w}_{t}\right)=0$.

Proposition 10 concludes the proof of Proposition 1: for the finite-horizon bounded economy $\mathcal{E}^{T},\left(\overline{\mathbf{p}}, \overline{\mathbf{r}}, \overline{\mathbf{w}},\left(\overline{\mathbf{c}}_{h}, \overline{\mathbf{k}}_{h}, \overline{\boldsymbol{\lambda}}_{h}\right)_{h=1}^{m}, \overline{\mathbf{K}}, \overline{\mathbf{L}}\right)$ is actually an equilibrium.

\section{Appendix 2: existence of equilibrium in an infinite-horizon economy}

We want to prove Theorem 5 . From now on, any variable $x_{t}^{T}$ with subscript $t$ and superscript $T$ will refer to period $t$ in a $T$-truncated economy with $x_{t}^{T}=0$ for $t>T$. As above, sequences will be denoted in bold type. 
Under the Assumptions 1, 2, 3 and 5, an equilibrium

$$
\left(\overline{\mathbf{p}}, \overline{\mathbf{r}}, \overline{\mathbf{w}},\left(\overline{\mathbf{c}}_{i}, \overline{\mathbf{k}}_{i}, \overline{\boldsymbol{\lambda}}_{i}\right)_{i=1}^{m}, \overline{\mathbf{K}}, \overline{\mathbf{L}}\right)^{T}
$$

of a truncated economy exists. Under these assumptions, namely separability and differentiability of preferences, the Kuhn-Tucker necessary conditions hold for the existence of an equilibrium in a truncated economy.

Claim 15 Under Assumption 5, the equilibrium of a truncated economy satisfies the following conditions.

For $t=0, \ldots, T$ :

(1) $\bar{p}_{t}^{T}, \bar{r}_{t}^{T}, \bar{w}_{t}^{T}>0$ with $\bar{p}_{t}^{T}+\bar{r}_{t}^{T}+\bar{w}_{t}^{T}=1$ (normalization),

(2) $\left(\partial F / \partial K_{t}\right)\left(\bar{K}_{t}^{T}, \bar{L}_{t}^{T}\right)=\bar{r}_{t}^{T} / \bar{p}_{t}^{T}$,

(3) $\left(\partial F / \partial L_{t}\right)\left(\bar{K}_{t}^{T}, \bar{L}_{t}^{T}\right)=\bar{w}_{t}^{T} / \bar{p}_{t}^{T}$,

(4) $\bar{K}_{t}^{T}=\sum_{i=1}^{m} \bar{k}_{i t}^{T}$,

(5) $\bar{L}_{t}^{T}=\sum_{i=1}^{m} \bar{l}_{i t}^{T}$,

(6) $\sum_{i=1}^{m}\left[\bar{c}_{i t}^{T}+\bar{k}_{i t+1}^{T}-(1-\delta) \bar{k}_{i t}^{T}\right]=F\left(\bar{K}_{t}^{T}, \bar{L}_{t}^{T}\right)$ with $\bar{k}_{i T+1}^{T}=0$.

For $i=1, \ldots, m, t=0, \ldots, T$ :

(7) $\beta_{i}^{t} u_{i}^{\prime}\left(\bar{c}_{i t}^{T}\right)=\bar{\mu}_{i t}^{T} \bar{p}_{t}^{T} \geq \bar{\mu}_{i t+1}^{T} \bar{p}_{t+1}^{T}(1-\delta)+\bar{\mu}_{i t+1}^{T} \bar{r}_{t+1}^{T}$, with equality when $\bar{k}_{i t+1}^{T}>0$,

(8) $v_{i}^{\prime}\left(\bar{\lambda}_{i t}^{T}\right) \geq u_{i}^{\prime}\left(\bar{c}_{i t}^{T}\right) \bar{w}_{t}^{T} / \bar{p}_{t}^{T}$, with equality when $\bar{\lambda}_{i t}^{T}<1$,

(9) $\bar{p}_{t}^{T}\left[\bar{c}_{i t}^{T}+\bar{k}_{i t+1}^{T}-(1-\delta) \bar{k}_{i t}^{T}\right]=\bar{r}_{t}^{T} \bar{k}_{i t}^{T}+\bar{w}_{t}^{T}\left(1-\bar{\lambda}_{i t}^{T}\right)$ with $\bar{k}_{i t}^{T} \geq 0, \bar{k}_{i T+1}^{T}=$ 0 and $0 \leq \bar{\lambda}_{i t}^{T} \leq 1$,

where $\bar{\mu}_{i t}^{T}$ is the multiplier associated to the budget constraint at time $t$.

Proof. See Bosi and Seegmuller (2010).

In the rest of Appendix 2, from Claim 16 to 26, we will prove that the equilibrium variables (prices and quantities) of the sequence of truncated economies converge to limit values that are actually equilibrium values of the limit economy. For simplicity, we will omit any reference to Assumptions 1, 2, 3 and 5. We will suppose that they are always satisfied.

Let us simplify the notation:

$$
\begin{array}{lllll}
\bar{\zeta}_{i t}^{T} \equiv \beta_{i}^{t} u_{i}^{\prime}\left(\bar{c}_{i t}^{T}\right) \bar{c}_{i t}^{T} & \text { if } t \leq T, & \text { and } & \bar{\zeta}_{i t}^{T}=0 & \text { if } t>T, \\
\bar{\eta}_{i t}^{T} \equiv \beta_{i}^{t} v_{i}^{\prime}\left(\bar{\lambda}_{i t}^{T}\right) \bar{\lambda}_{i t}^{T} & \text { if } t \leq T, & \text { and } & \bar{\eta}_{i t}^{T}=0 & \text { if } t>T, \\
\bar{\theta}_{i t}^{T} \equiv \beta_{i}^{t} v_{i}^{\prime}\left(\bar{\lambda}_{i t}^{T}\right) & \text { if } t \leq T, & \text { and } & \bar{\theta}_{i t}^{T}=0 & \text { if } t>T, \\
\bar{\vartheta}_{i t}^{T} \equiv \bar{\mu}_{i t}^{T} \bar{w}_{t}^{T} & \text { if } t \leq T, & \text { and } & \bar{\vartheta}_{i t}^{T}=0 & \text { if } t>T,
\end{array}
$$

and $\bar{\varepsilon}_{i t}^{T} \equiv \bar{\theta}_{i t}^{T}-\bar{\vartheta}_{i t}^{T}$.

We notice that points (7) and (8) of Claim 15 entail $\bar{\varepsilon}_{i t}^{T} \geq 0$ with $\bar{\varepsilon}_{i t}^{T}=0$ when $\bar{\lambda}_{i t}^{T}<1$.

Claim 16 For any $\varepsilon>0$, there exists $\tau$ such that, for any $s>\tau$ and any $T$, $\sum_{t=s}^{\infty} \bar{\zeta}_{i t}^{T}<\varepsilon$. 
We observe that the critical $\tau$ is independent of $T$.

Proof. We know that, under Assumptions 1 and $2, \bar{k}_{i t}^{T} \leq A$ and $\bar{c}_{i t}^{T} \leq A$. We observe that $\sum_{t=0}^{\infty} \beta_{i}^{t} u_{i}(A)=u_{i}(A) /\left(1-\beta_{i}\right)<\infty$. Then, there exists $\tau$ such that $\sum_{t=\tau}^{\infty} \beta_{i}^{t} u_{i}(A)<\varepsilon$. In addition, under Assumption 5,

$$
\begin{aligned}
\sum_{t=\tau}^{\infty} \beta_{i}^{t} u_{i}(A) & \geq \sum_{t=\tau}^{T} \beta_{i}^{t} u_{i}\left(\bar{c}_{i t}^{T}\right)=\sum_{t=\tau}^{T} \beta_{i}^{t}\left[u_{i}\left(\bar{c}_{i t}^{T}\right)-u_{i}(0)\right] \\
& \geq \sum_{t=\tau}^{T} \beta_{i}^{t} u_{i}^{\prime}\left(\bar{c}_{i t}^{T}\right) \bar{c}_{i t}^{T}
\end{aligned}
$$

because of the concavity of $u_{i}$. Thus, for any $\varepsilon>0$, there exists $\tau$ such that, for any $s>\tau$ and any $T, \sum_{t=s}^{\infty} \bar{\zeta}_{i t}^{T}<\varepsilon$.

Claim 17 For any $\varepsilon>0$, there exists $\tau$ such that, for any $s>\tau$ and any $T$, $\sum_{t=s}^{\infty} \bar{\eta}_{i t}^{T}<\varepsilon$.

As above, the critical $\tau$ does not depend on $T$.

Proof. Since $\sum_{t=0}^{\infty} \beta_{i}^{t} v_{i}(1)=v_{i}(1) /\left(1-\beta_{i}\right)<\infty$, there exists $\tau$ such that $\sum_{t=\tau}^{\infty} \beta_{i}^{t} v_{i}(1)<\varepsilon$. In addition, under Assumption 5 ,

$$
\begin{aligned}
\sum_{t=\tau}^{\infty} \beta_{i}^{t} v_{i}(1) & \geq \sum_{t=\tau}^{T} \beta_{i}^{t} v_{i}\left(\bar{\lambda}_{i t}^{T}\right)=\sum_{t=\tau}^{T} \beta_{i}^{t}\left[v_{i}\left(\bar{\lambda}_{i t}^{T}\right)-v_{i}(0)\right] \\
& \geq \sum_{t=\tau}^{T} \beta_{i}^{t} v_{i}^{\prime}\left(\bar{\lambda}_{i t}^{T}\right) \bar{\lambda}_{i t}^{T}
\end{aligned}
$$

because $\bar{\lambda}_{i t}^{T} \leq 1$ and $v_{i}$ is concave. Thus, for any $\varepsilon>0$, there exists $\tau$ such that, for any $s>\tau$ and any $T, \sum_{t=s}^{\infty} \bar{\eta}_{i t}^{T}<\varepsilon$.

Claim 18 For any $\varepsilon>0$, there exists $\tau$ such that, for any $s>\tau$ and any $T$, $\sum_{t=s}^{\infty} \bar{\vartheta}_{i t}^{T} \bar{\lambda}_{i t}^{T}<\varepsilon$ and $\sum_{t=s}^{\infty} \bar{\varepsilon}_{i t}^{T}<\varepsilon$. In addition, for any $T,\left(\bar{\vartheta}_{i t}^{T} \bar{\lambda}_{i t}^{T}\right)_{t=0}^{\infty} \in l_{+}^{1}$ and $\left(\bar{\varepsilon}_{i t}^{T}\right)_{t=0}^{\infty} \in l_{+}^{1}$.

Notice that the critical $\tau$ does not depend on $T$.

Proof. From (29), we observe that $\beta_{i}^{t} v_{i}^{\prime}\left(\bar{\lambda}_{i t}^{T}\right) \bar{\lambda}_{i t}^{T}=\bar{\vartheta}_{i t}^{T} \bar{\lambda}_{i t}^{T}+\bar{\varepsilon}_{i t}^{T} \bar{\lambda}_{i t}^{T}=\bar{\vartheta}_{i t}^{T} \bar{\lambda}_{i t}^{T}+\bar{\varepsilon}_{i t}^{T}$ since $\bar{\varepsilon}_{i t}^{T}=0$ when $\bar{\lambda}_{i t}^{T}<1$. For any $\varepsilon>0$, there exists $\tau$ such that, for any $s>\tau$, $\sum_{t=s}^{\infty} \beta_{i}^{t} v_{i}(1)<\varepsilon$. Thus, according to (31), for any $\varepsilon>0$, there exists $\tau$ such that, for any $s>\tau$ and any $T, \sum_{t=s}^{T}\left(\bar{\vartheta}_{i t}^{T} \bar{\lambda}_{i t}^{T}+\bar{\varepsilon}_{i t}^{T}\right)=\sum_{t=s}^{T} \beta_{i}^{t} v_{i}^{\prime}\left(\bar{\lambda}_{i t}^{T}\right) \bar{\lambda}_{i t}^{T}<\varepsilon$. In particular, $\sum_{t=s}^{\infty} \bar{\vartheta}_{i t}^{T} \bar{\lambda}_{i t}^{T}<\varepsilon$ and $\sum_{t=s}^{\infty} \bar{\varepsilon}_{i t}^{T}<\varepsilon$.

From (31), we have also, for any $T, \sum_{t=0}^{\infty}\left(\bar{\vartheta}_{i t}^{T} \bar{\lambda}_{i t}^{T}+\bar{\varepsilon}_{i t}^{T}\right) \leq \sum_{t=0}^{\infty} \beta_{i}^{t} v_{i}(1)=$ $v_{i}(1) /\left(1-\beta_{i}\right)$ and, so, $\sum_{t=0}^{\infty} \bar{\vartheta}_{i t}^{T} \bar{\lambda}_{i t}^{T} \leq v_{i}(1) /\left(1-\beta_{i}\right)$ and

$$
\sum_{t=0}^{\infty} \bar{\varepsilon}_{i t}^{T} \leq v_{i}(1) /\left(1-\beta_{i}\right)
$$


Then, for any $T,\left(\bar{\vartheta}_{i t}^{T} \bar{\lambda}_{i t}^{T}\right)_{t=0}^{\infty} \in l_{+}^{1}$ and $\left(\bar{\varepsilon}_{i t}^{T}\right)_{t=0}^{\infty} \in l_{+}^{1}$.

Claim 19 For any $\varepsilon>0$, there exists $\tau$ such that for any $s>\tau$ and any $T \geq s$ we have $\sum_{t=s}^{T} \bar{\vartheta}_{i t}^{T}<\varepsilon$. In addition, for any $T$,

$$
\sum_{t=0}^{T} \bar{\vartheta}_{i t}^{T}<\frac{u_{i}(A)+v_{i}(1)}{1-\beta_{i}}
$$

Proof. Focus now on the sequence of equilibrium budget constraints: $\bar{r}_{t}^{T} \bar{k}_{i t}^{T}+$ $\bar{w}_{t}^{T}\left(1-\bar{\lambda}_{i t}^{T}\right)-\bar{p}_{t}^{T}\left[\bar{c}_{i t}^{T}+\bar{k}_{i t+1}^{T}-(1-\delta) \bar{k}_{i t}^{T}\right] \geq 0$.

Multiplying them by the multipliers, we obtain, according to the KuhnTucker method,

$$
\bar{\mu}_{i t}^{T} \bar{r}_{t}^{T} \bar{k}_{i t}^{T}+\bar{\mu}_{i t}^{T} \bar{w}_{t}^{T}\left(1-\bar{\lambda}_{i t}^{T}\right)-\bar{\mu}_{i t}^{T} \bar{p}_{t}^{T} \bar{c}_{i t}^{T}-\bar{\mu}_{i t}^{T} \bar{p}_{t}^{T} \bar{k}_{i t+1}^{T}+\bar{\mu}_{i t}^{T} \bar{p}_{t}^{T}(1-\delta) \bar{k}_{i t}^{T}=0
$$

Summing them over time from $\tau$ to $T$, we get

$$
\begin{aligned}
& \bar{\mu}_{i \tau}^{T} \bar{r}_{\tau}^{T} \bar{k}_{i \tau}^{T}+\bar{\mu}_{i \tau}^{T} \bar{w}_{\tau}^{T}\left(1-\bar{\lambda}_{i \tau}^{T}\right)-\bar{\mu}_{i \tau}^{T} \bar{p}_{\tau}^{T} \bar{c}_{i \tau}^{T}-\bar{\mu}_{i \tau}^{T} \bar{p}_{\tau}^{T} \bar{k}_{i \tau+1}^{T}+\bar{\mu}_{i \tau}^{T} \bar{p}_{\tau}^{T}(1-\delta) k_{i \tau} \\
& +\bar{\mu}_{i \tau+1}^{T} \bar{r}_{\tau+1}^{T} \bar{k}_{i \tau+1}^{T}+\bar{\mu}_{i \tau+1}^{T} \bar{w}_{\tau+1}^{T}\left(1-\bar{\lambda}_{i \tau+1}^{T}\right)-\bar{\mu}_{i \tau+1}^{T} \bar{p}_{\tau+1}^{T} \bar{c}_{i \tau+1}^{T} \\
& -\bar{\mu}_{i \tau+1}^{T} \bar{p}_{\tau+1}^{T} \bar{k}_{i \tau+2}^{T}+\bar{\mu}_{i \tau+1}^{T} \bar{p}_{\tau+1}^{T}(1-\delta) \bar{k}_{i \tau+1}^{T} \\
& +\ldots \\
& +\bar{\mu}_{i T}^{T} \bar{r}_{T}^{T} \bar{k}_{i T}^{T}+\bar{\mu}_{i T}^{T} \bar{w}_{T}^{T}\left(1-\bar{\lambda}_{i T}^{T}\right)-\bar{\mu}_{i T}^{T} \bar{p}_{T}^{T} \bar{c}_{i T}^{T}-\bar{\mu}_{i T}^{T} \bar{p}_{T}^{T} \bar{k}_{i T+1}^{T} \\
& +\bar{\mu}_{i T}^{T} \bar{p}_{T}^{T}(1-\delta) \bar{k}_{i T}^{T} \\
= & 0
\end{aligned}
$$

that is

$$
\begin{aligned}
& \sum_{t=\tau}^{T} \bar{\vartheta}_{i t}^{T}-\sum_{t=\tau}^{T} \bar{\vartheta}_{i t}^{T} \bar{\lambda}_{i t}^{T} \\
& -\sum_{t=\tau}^{T-1}\left[\bar{\mu}_{i t}^{T} \bar{p}_{t}^{T}-\bar{\mu}_{i t+1}^{T} \bar{p}_{t+1}^{T}(1-\delta)-\bar{\mu}_{i t+1}^{T} \bar{r}_{t+1}^{T}\right] \bar{k}_{i t+1}^{T} \\
& +\bar{\mu}_{i \tau}^{T} \bar{p}_{\tau}^{T}(1-\delta) k_{i \tau}+\bar{\mu}_{i \tau}^{T} \bar{r}_{\tau}^{T} k_{i \tau}-\bar{\mu}_{i T}^{T} \bar{p}_{T}^{T} \bar{k}_{i T+1}^{T} \\
= & \sum_{t=\tau}^{T} \bar{\mu}_{i t}^{T} \bar{p}_{t}^{T} \bar{c}_{i t}^{T}=\sum_{t=\tau}^{T} \beta_{i}^{t} u_{i}^{\prime}\left(\bar{c}_{i t}^{T}\right) \bar{c}_{i t}^{T}=\sum_{t=\tau}^{T} \bar{\zeta}_{i t}^{T}
\end{aligned}
$$

We know that $\left[\bar{\mu}_{i t}^{T} \bar{p}_{t}^{T}-\bar{\mu}_{i t+1}^{T} \bar{p}_{t+1}^{T}(1-\delta)-\bar{\mu}_{i t \pm 1}^{T} \bar{r}_{t+1}^{T}\right] \bar{k}_{i t+1}^{T}=0$ because either $\bar{\mu}_{i t}^{T} \bar{p}_{t}^{T}-\bar{\mu}_{i t+1}^{T} \bar{p}_{t+1}^{T}(1-\delta)-\bar{\mu}_{i t+1}^{T} \bar{r}_{t+1}^{T}=0$ or $\bar{k}_{i t+1}^{T}=0$ (point (7) of Claim 
15). Then

$$
\begin{aligned}
\sum_{t=\tau}^{T} \bar{\vartheta}_{i t}^{T}= & \sum_{t=\tau}^{T} \bar{\zeta}_{i t}^{T}+\sum_{t=\tau}^{T} \bar{\vartheta}_{i t}^{T} \bar{\lambda}_{i t}^{T} \\
& -\bar{\mu}_{i \tau}^{T} \bar{p}_{\tau}^{T}(1-\delta) k_{i \tau}-\bar{\mu}_{i \tau}^{T} \bar{r}_{\tau}^{T} k_{i \tau}+\bar{\mu}_{i T}^{T} \bar{p}_{T}^{T} \bar{k}_{i T+1}^{T}
\end{aligned}
$$

From the proof of Claim 16, we know that

$$
\sum_{t=\tau}^{T} \bar{\zeta}_{i t}^{T} \leq \sum_{t=\tau}^{T} \beta_{i}^{t} u_{i}(A)=u_{i}(A) \frac{\beta_{i}^{\tau}-\beta_{i}^{T+1}}{1-\beta_{i}}<\frac{\beta_{i}^{\tau} u_{i}(A)}{1-\beta_{i}}
$$

Thus, for any $\varepsilon>0$, there exists $\tau_{1}$ such that, for any $s>\tau_{1}$ and any $T \geq s$,

$$
\sum_{t=s}^{T} \bar{\zeta}_{i t}^{T}<\varepsilon / 2
$$

From the proof of Claim 18, we know also that

$$
\sum_{t=\tau}^{T} \bar{\vartheta}_{i t}^{T} \bar{\lambda}_{i t}^{T} \leq \sum_{t=\tau}^{T} \beta_{i}^{t} v_{i}(1)=v_{i}(1) \frac{\beta_{i}^{\tau}-\beta_{i}^{T+1}}{1-\beta_{i}}<\frac{\beta_{i}^{\tau} v_{i}(1)}{1-\beta_{i}}
$$

Thus, for any $\varepsilon>0$, there exists $\tau_{2}$ such that, for any $s>\tau_{2}$ and any $T \geq s$, $\sum_{t=s}^{T} \bar{\vartheta}_{i t}^{T} \bar{\lambda}_{i t}^{T}<\varepsilon / 2$. According to (34), we have that

$$
\begin{aligned}
\sum_{t=s}^{T} \bar{\vartheta}_{i t}^{T} & \leq \sum_{t=s}^{T} \bar{\zeta}_{i t}^{T}+\sum_{t=s}^{T} \bar{\vartheta}_{i t}^{T} \bar{\lambda}_{i t}^{T}+\bar{\mu}_{i T}^{T} \bar{p}_{T}^{T} \bar{k}_{i T+1}^{T} \\
& =\sum_{t=s}^{T} \bar{\zeta}_{i t}^{T}+\sum_{t=s}^{T} \bar{\vartheta}_{i t}^{T} \bar{\lambda}_{i t}^{T}
\end{aligned}
$$

because in the truncated economy $\bar{k}_{i T+1}^{T}=0$.

Thus, for any $\varepsilon>0$, there exists $\tau \equiv \max \left\{\tau_{1}, \tau_{2}\right\}$ such that, for any $s>\tau$ and any $T \geq s, \sum_{t=s}^{T} \bar{\vartheta}_{i t}^{T}<\varepsilon / 2+\varepsilon / 2=\varepsilon$ because in the truncated economy $\bar{k}_{i T+1}^{T}=0$.

Finally, from (35), (37) and (38), we have

$$
\sum_{t=\tau}^{T} \bar{\vartheta}_{i t}^{T} \leq \sum_{t=\tau}^{T} \bar{\zeta}_{i t}^{T}+\sum_{t=\tau}^{T} \bar{\vartheta}_{i t}^{T} \bar{\lambda}_{i t}^{T}<\left[u_{i}(A)+v_{i}(1)\right] \frac{\beta_{i}^{\tau}}{1-\beta_{i}}
$$

Taking $\tau=0$, we obtain (32).

Claim 20 Let $\overline{\boldsymbol{\vartheta}}_{i}^{T} \equiv\left(\bar{\vartheta}_{i t}^{T}\right)_{t=0}^{\infty}$. There is a subsequence $\left(\overline{\boldsymbol{\vartheta}}_{i}^{T_{S}}\right)_{S=0}^{\infty}$ which converges for the $l^{1}$-topology to a sequence $\overline{\boldsymbol{\vartheta}}_{i} \equiv\left(\bar{\vartheta}_{i t}\right)_{t=0}^{\infty} \in l_{+}^{1}$. The limit $\overline{\boldsymbol{\vartheta}}_{i}$ shares the same properties of the terms $\overline{\boldsymbol{\vartheta}}_{i}^{T}$ of the sequence, namely, (1) for any $\varepsilon>0$ there exists $\tau$ (the same for all the terms) such that, for any $s>\tau$, we have $\sum_{t=s}^{\infty} \bar{\vartheta}_{i t} \leq \varepsilon$, and (2) $\sum_{t=0}^{\infty} \bar{\vartheta}_{i t} \leq\left[u_{i}(A)+v_{i}(1)\right] /\left(1-\beta_{i}\right)$. 
Proof. We apply Claim 19 and we find that, for any $\varepsilon>0$ there exists $\tau$ such that for any $s>\tau$ and any $T$, we have $\sum_{t=s}^{\infty} \bar{\vartheta}_{i t}^{T} \leq \varepsilon$. We observe also that (32) implies $\sum_{t=0}^{\infty} \bar{\vartheta}_{i t}^{T} \leq\left[u_{i}(A)+v_{i}(1)\right] /\left(1-\beta_{i}\right)$ for any $T$. Thus, Lemma 4 in Appendix 3 applies with a ball $B$ of radius $\rho=\left[u_{i}(A)+v_{i}(1)\right] /\left(1-\beta_{i}\right)$.

Claim 21 In the infinite-horizon economy, leisure demand is positive:

$$
\lim _{T \rightarrow \infty} \bar{\lambda}_{i t}^{T}=\bar{\lambda}_{i t} \in(0,1]
$$

Proof. We have $\bar{\theta}_{i t}^{T}=\bar{\vartheta}_{i t}^{T}+\bar{\varepsilon}_{i t}^{T}$ with $\bar{\varepsilon}_{i t}^{T} \geq 0$ and $\bar{\varepsilon}_{i t}^{T}=0$ if $\bar{\lambda}_{i t}^{T}<1$.

From Claim 19, we know that, for any $\varepsilon>0$, there exists $\tau_{1}$ such that, for any $s>\tau_{1}$ and any $T, \sum_{t=s}^{\infty} \bar{\vartheta}_{i t}^{T} \leq \varepsilon / 2$.

From Claim 18, we know that for any $\varepsilon>0$, there exists $\tau_{2}$ such that, for any $s>\tau_{2}$ and any $T, \sum_{t=s}^{\infty} \bar{\varepsilon}_{i t}^{T}<\varepsilon / 2$.

Hence, for any $\varepsilon>0$, there exists $\tau \equiv \max \left\{\tau_{1}, \tau_{2}\right\}$ such that, for any $s>\tau$ and any $T, \sum_{t=s}^{\infty} \bar{\theta}_{i t}^{T}=\sum_{t=s}^{\infty} \bar{\vartheta}_{i t}^{T}+\sum_{t=s}^{\infty} \bar{\varepsilon}_{i t}^{T}<\varepsilon$. In addition, for any $T$,

$$
\sum_{t=0}^{\infty} \bar{\theta}_{i t}^{T}=\sum_{t=0}^{\infty} \bar{\vartheta}_{i t}^{T}+\sum_{t=0}^{\infty} \bar{\varepsilon}_{i t}^{T} \leq \frac{u_{i}(A)+v_{i}(1)}{1-\beta_{i}}+\frac{v_{i}(1)}{1-\beta_{i}}
$$

Let $\overline{\boldsymbol{\theta}}_{i}^{T} \equiv\left(\bar{\theta}_{i t}^{T}\right)$. Then $\overline{\boldsymbol{\theta}}_{i}^{T} \rightarrow \overline{\boldsymbol{\theta}}_{i} \in l_{+}^{1}$ for the $l^{1}$-topology (Lemma 4 in Appendix 3 applies with $\left.\rho=\left[u_{i}(A)+2 v_{i}(1)\right] /\left(1-\beta_{i}\right)\right)$.

Therefore, for any $t, \bar{\theta}_{i t}^{T}$ converges to $\bar{\theta}_{i t} \in(0,+\infty)$. Hence, $\bar{\lambda}_{i t}^{T}$ converges to $\bar{\lambda}_{i t}>0$ since $v_{i}$ satisfies the Inada conditions (Assumption 5). Clearly, $\bar{\lambda}_{i t} \leq 1$.

Claim 22 In the infinite-horizon economy, the equilibrium prices are positive: $\lim _{T \rightarrow \infty} \bar{p}_{t}^{T}=\bar{p}_{t} \in(0,1), \lim _{T \rightarrow \infty} \bar{r}_{t}^{T}=\bar{r}_{t} \in(0,1), \lim _{T \rightarrow \infty} \bar{w}_{t}=\bar{w}_{t} \in(0,1)$.

Proof. Focus on prices.

Suppose that $\lim _{T \rightarrow \infty} \bar{p}_{t}^{T}=0$. We know that $\beta_{i}^{t} u_{i}^{\prime}\left(\bar{c}_{i t}^{T}\right)=\bar{\mu}_{i t}^{T} \bar{p}_{t}^{T}$.

If $\bar{\mu}_{i t}^{T}$ is bounded, we have $\lim _{T \rightarrow \infty} u_{i}^{\prime}\left(\bar{c}_{i t}^{T}\right)=0$ which is impossible because $\bar{c}_{i t}^{T} \leq A$ for every $T$.

Then, $\lim _{T \rightarrow \infty} \bar{\mu}_{i t}^{T}=+\infty$. However, $\beta_{i}^{t} v_{i}^{\prime}\left(\bar{\lambda}_{i t}^{T}\right) / \bar{\mu}_{i t}^{T}=\bar{w}_{t}^{T}+\bar{\varepsilon}_{i t}^{T} / \bar{\mu}_{i t}^{T}$ and $\lim _{T \rightarrow \infty} \bar{w}_{t}^{T}=\lim _{T \rightarrow \infty}\left(\bar{\theta}_{i t}^{T} / \bar{\mu}_{i t}^{T}\right)-\lim _{T \rightarrow \infty}\left(\bar{\varepsilon}_{i t}^{T} / \bar{\mu}_{i t}^{T}\right)=0$ (Claim 21).

Since $\lim _{T \rightarrow \infty} \bar{p}_{t}^{T}=0, \lim _{T \rightarrow \infty} \bar{w}_{t}^{T}=0$ and $\bar{p}_{t}^{T}+\bar{w}_{t}^{T}+\bar{r}_{t}^{T}=1$, we get $\lim _{T \rightarrow \infty} \bar{r}_{t}^{T}=1$.

We know that $\bar{\mu}_{i t-1}^{T} \bar{p}_{t-1}^{T} \geq \bar{\mu}_{i t}^{T} \bar{p}_{t}^{T}(1-\delta)+\bar{\mu}_{i t}^{T} \bar{r}_{t}^{T} \geq \bar{\mu}_{i t}^{T} \bar{r}_{t}^{T}$ (point (7) of Claim 15). Then $\lim _{T \rightarrow \infty} \bar{\mu}_{i t-1}^{T} \bar{p}_{t-1}^{T} \geq \lim _{T \rightarrow \infty} \bar{\mu}_{i t}^{T} \bar{r}_{t}^{T}=+\infty$.

Similarly, $\bar{\mu}_{i t-2}^{T} \bar{p}_{t-2}^{T} \geq \bar{\mu}_{i t-1}^{T} \bar{p}_{t-1}^{T}(1-\delta)+\bar{\mu}_{i t-1}^{T} \bar{r}_{t-1}^{T} \geq \bar{\mu}_{i t-1}^{T} \bar{p}_{t-1}^{T}(1-\delta)$ and $\lim _{T \rightarrow \infty} \bar{\mu}_{i t-2}^{T} \bar{p}_{t-2}^{T} \geq \lim _{T \rightarrow \infty} \bar{\mu}_{i t-1}^{T} \bar{p}_{t-1}^{T}(1-\delta)=+\infty$.

Computing backward, we obtain $\lim _{T \rightarrow \infty} \bar{\mu}_{i 0}^{T} \bar{p}_{0}^{T}=+\infty$. 
If $\lim _{T \rightarrow \infty} \bar{p}_{0}^{T}>0$, since $\bar{p}_{0}^{T} \leq 1$, then $\lim _{T \rightarrow \infty} \bar{\mu}_{i 0}^{T}=+\infty$ and, since $\lim _{T \rightarrow \infty} \bar{\mu}_{i 0}^{T} \bar{w}_{0}^{T}=\bar{\vartheta}_{i 0}<+\infty$, this implies $\lim _{T \rightarrow \infty} \bar{w}_{0}^{T}=0$. Thus,

$$
0=\bar{p}_{0} F\left(K_{0}, \bar{L}_{0}\right)-\bar{r}_{0} K_{0}-\bar{w}_{0} \bar{L}_{0}=\bar{p}_{0} F\left(K_{0}, \bar{L}_{0}\right)-\bar{r}_{0} K_{0}
$$

Choose $L_{0}>\bar{L}_{0}$ in order to obtain a higher profit in contradiction with profit maximization.

Let $\lim _{T \rightarrow \infty} \bar{p}_{0}^{T}=0$. We know that $u_{i}^{\prime}(A) \leq u_{i}^{\prime}\left(\bar{c}_{i 0}^{T}\right)=\beta_{i}^{0} u_{i}^{\prime}\left(\bar{c}_{i 0}^{T}\right)=\bar{\mu}_{i 0}^{T} \bar{p}_{0}^{T}$.

If $\lim _{T \rightarrow \infty} \bar{\mu}_{i 0}^{T}<+\infty$, we have $\lim _{T \rightarrow \infty} \bar{\mu}_{i 0}^{T} \bar{p}_{0}^{T}=0$ and $u_{i}^{\prime}(A) \leq 0$, a contradiction.

If $\lim _{T \rightarrow \infty} \bar{\mu}_{i 0}^{T}=+\infty$, then $\lim _{T \rightarrow \infty} \bar{\mu}_{i 0}^{T} \bar{w}_{0}^{T}=\bar{\vartheta}_{i 0}<+\infty$ gives $\lim _{T \rightarrow \infty} \bar{w}_{0}^{T}=$ 0 and $\lim _{T \rightarrow \infty} \bar{r}_{0}^{T}=1$. Focus on the first budget constraint:

$$
\bar{p}_{0}^{T}\left[\bar{c}_{i 0}^{T}+\bar{k}_{i 1}^{T}-(1-\delta) k_{i 0}\right]=\bar{r}_{0}^{T} k_{i 0}+\bar{w}_{0}^{T}\left(1-\bar{\lambda}_{i 0}^{T}\right)
$$

Assumption 3 ensures $k_{i 0}>0$. In this case, in the limit:

$$
0=\bar{p}_{0}\left[\bar{c}_{i 0}+\bar{k}_{i 1}-(1-\delta) k_{i 0}\right]=\bar{r}_{0} k_{i 0}+\bar{w}_{0}\left(1-\bar{\lambda}_{i 0}\right) \geq k_{i 0}>0
$$

a contradiction. Thus, for every $t, \bar{p}_{t}^{T} \rightarrow \bar{p}_{t}>0$.

Focus now on $\bar{r}_{t}$ and $\bar{w}_{t}$. In the limit, $\bar{p}_{t} F\left(\bar{K}_{t}, \bar{L}_{t}\right)-\bar{r}_{t} \bar{K}_{t}-\bar{w}_{t} \bar{L}_{t}=0$.

If $\bar{r}_{t}=0$ (respectively, $\bar{w}_{t}=0$ ), then $\bar{p}_{t} F\left(\bar{K}_{t}, \bar{L}_{t}\right)-\bar{w}_{t} \bar{L}_{t}=0$ (respectively, $\left.\bar{p}_{t} F\left(\bar{K}_{t}, \bar{L}_{t}\right)-\bar{r}_{t} \bar{K}_{t}=0\right)$. Fix $L_{t}>0\left(K_{t}>0\right)$ and choose $K_{t}\left(L_{t}\right)$ large enough such that $\bar{p}_{t} F\left(K_{t}, L_{t}\right)-\bar{w}_{t} L_{t}>0\left(\bar{p}_{t} F\left(K_{t}, L_{t}\right)-\bar{r}_{t} K_{t}>0\right)$, against the equilibrium condition.

Thus, $\bar{p}_{t}, \bar{r}_{t}, \bar{w}_{t}>0$.

Claim $23 \bar{c}_{i t}=\lim _{T \rightarrow \infty} \bar{c}_{i t}^{T} \in(0,+\infty)$.

Proof. For any $t, \sum_{i=1}^{m} \bar{c}_{i t}^{T} \leq A$. This implies $\bar{c}_{i t}^{T} \leq A$ independently of the choice of $T$ and $\lim _{T \rightarrow \infty} \bar{c}_{i t}^{T} \leq A<+\infty$. In addition, if $\bar{c}_{i t}=\lim _{T \rightarrow \infty} \bar{c}_{i t}^{T}=0$, then, since $u_{i}^{\prime}\left(\bar{c}_{i t}^{T}\right) \bar{w}_{t}^{T} / \bar{p}_{t}^{T} \leq v_{i}^{\prime}\left(\bar{\lambda}_{i t}^{T}\right)$, we obtain $+\infty=\lim _{T \rightarrow \infty} u_{i}^{\prime}\left(\bar{c}_{i t}^{T}\right) \bar{w}_{t}^{T} / \bar{p}_{t}^{T} \leq$ $\lim _{T \rightarrow \infty} v_{i}^{\prime}\left(\bar{\lambda}_{i t}^{T}\right)$, that is $\bar{\lambda}_{i t}=\lim _{T \rightarrow \infty} \bar{\lambda}_{i t}^{T}=0$, a contradiction (see Claim 21). Then, $\bar{c}_{i t}>0$.

Claim 24 For any $t, \lim _{T \rightarrow \infty} \bar{K}_{t}^{T}=\bar{K}_{t}>0$ and $\lim _{T \rightarrow \infty} \bar{L}_{t}^{T}=\bar{L}_{t}>0$.

Proof. We know that $\sum_{i=1}^{m} \bar{k}_{i t+1} \geq 0$ and that $\sum_{i=1}^{m} \bar{c}_{i t}+\sum_{i=1}^{m} \bar{k}_{i t+1}=$ $F\left(\bar{K}_{t}, \bar{L}_{t}\right)+(1-\delta) \bar{K}_{t}$. If $\overline{\bar{K}}_{t}=0$, then $\bar{c}_{i t}=0$ for every $i$, a contradiction. Now, if $\bar{L}_{t}=0$, we have $\bar{r}_{t} \bar{K}_{t}=0$ and, hence, $\bar{K}_{t}=0$ : a contradiction.

The transversality condition holds.

Claim $25 \lim _{t \rightarrow+\infty} \bar{\mu}_{i t} \bar{p}_{t} \bar{k}_{i t+1}=0$.

Proof. Let $\varepsilon>0$. We know that there exists $\tau$ such that for any pair $\left(s, s^{\prime}\right)$ such that $s^{\prime}>s>\tau$, and any $T>s$, we have $\sum_{t=s}^{s^{\prime}} \bar{\zeta}_{i t}^{T}<\varepsilon$ and $\sum_{t=s}^{s^{\prime}} \bar{\vartheta}_{i t}^{T}\left(1-\bar{\lambda}_{i t}^{T}\right)<$ 
$\varepsilon$ for every $i$ (inequality (36) and Claim 19). Taking the limit for $T \rightarrow+\infty$, we find

$$
\begin{aligned}
\varepsilon & \geq \lim _{T \rightarrow+\infty} \sum_{t=s}^{s^{\prime}} \bar{\zeta}_{i t}^{T}=\sum_{t=s}^{s^{\prime}} \lim _{T \rightarrow+\infty}\left[\beta_{i}^{t} u_{i}^{\prime}\left(\bar{c}_{i t}^{T}\right) \bar{c}_{i t}^{T}\right]=\sum_{t=s}^{s^{\prime}} \beta_{i}^{t} u_{i}^{\prime}\left(\bar{c}_{i t}\right) \bar{c}_{i t} \\
& =\sum_{t=s}^{s^{\prime}} \bar{\mu}_{i t} \bar{p}_{t} \bar{c}_{i t}
\end{aligned}
$$

(see Claim 23) and

$$
\begin{aligned}
\varepsilon & \geq \lim _{T \rightarrow+\infty} \sum_{t=s}^{s^{\prime}} \bar{\vartheta}_{i t}^{T}\left(1-\bar{\lambda}_{i t}^{T}\right)=\sum_{t=s}^{s^{\prime}} \lim _{T \rightarrow+\infty}\left(\bar{\mu}_{i t}^{T} \bar{w}_{t}^{T}\right)\left(1-\lim _{T \rightarrow+\infty} \bar{\lambda}_{i t}^{T}\right) \\
& =\sum_{t=s}^{s^{\prime}} \bar{\mu}_{i t} \bar{w}_{t}\left(1-\bar{\lambda}_{i t}\right)
\end{aligned}
$$

(see Claims 20 and 21). Since this holds for any $s^{\prime}>s$, we get also

$$
\sum_{t=s}^{\infty} \bar{\mu}_{i t} \bar{p}_{t} \bar{c}_{i t} \leq \varepsilon \text { and } \sum_{t=s}^{\infty} \bar{\mu}_{i t} \bar{w}_{t}\left(1-\bar{\lambda}_{i t}\right) \leq \varepsilon
$$

From the budget constraints, for any $s^{\prime} \geq T$, we obtain

$$
\begin{aligned}
\varepsilon & >\sum_{t=s}^{s^{\prime}} \bar{\mu}_{i t}^{T} \bar{p}_{t}^{T} \bar{c}_{i t}^{T}=\bar{\mu}_{i s}^{T} \bar{p}_{s}^{T}(1-\delta) \bar{k}_{i s}^{T}+\bar{\mu}_{i s}^{T} \bar{r}_{s}^{T} \bar{k}_{i s}^{T}+\sum_{t=s}^{s^{\prime}} \bar{\vartheta}_{i t}^{T}\left(1-\bar{\lambda}_{i t}^{T}\right) \\
& \geq \bar{\mu}_{i s}^{T} \bar{p}_{s}^{T}(1-\delta) \bar{k}_{i s}^{T}+\bar{\mu}_{i s}^{T} \bar{r}_{s}^{T} \bar{k}_{i s}^{T}
\end{aligned}
$$

(see (34)). Taking the limit for $T \rightarrow+\infty$, we find $\bar{\mu}_{i s} \bar{p}_{s}(1-\delta) \bar{k}_{i s} \leq \varepsilon$ and $\bar{\mu}_{i s} \bar{r}_{s} \bar{k}_{i s} \leq \varepsilon$ for every $s>\tau$. This implies $\lim \sup _{s} \bar{\mu}_{i s} \bar{p}_{s}(1-\delta) \bar{k}_{i s} \leq \varepsilon$ and $\lim \sup _{s} \bar{\mu}_{i s} \bar{r}_{s} \bar{k}_{i s} \leq \varepsilon$. These inequalities hold for any $\varepsilon>0$. Hence

$$
\lim _{t \rightarrow+\infty} \bar{\mu}_{i t} \bar{p}_{t}(1-\delta) \bar{k}_{i t}=0 \text { and } \lim _{t \rightarrow+\infty} \bar{\mu}_{i t} \bar{r}_{t} \bar{k}_{i t}=0
$$

Again, from the budget constraint, we have $\bar{\mu}_{i t}^{T} \bar{p}_{t}^{T} \bar{k}_{i t+1}^{T}=\bar{\mu}_{i t}^{T} \bar{p}_{t}^{T}(1-\delta) \bar{k}_{i t}^{T}+$ $\bar{\mu}_{i t}^{T} \bar{r}_{t}^{T} \bar{k}_{i t}^{T}+\bar{\mu}_{i t}^{T} \bar{w}_{t}^{T}\left(1-\bar{\lambda}_{i t}^{T}\right)-\bar{\mu}_{i t}^{T} \bar{p}_{t}^{T} \bar{c}_{i t}^{T}$ (see (33)). Taking the limit for $T \rightarrow+\infty$, we obtain $\bar{\mu}_{i t} \bar{p}_{t} \bar{k}_{i t+1}=\bar{\mu}_{i t} \bar{p}_{t}(1-\delta) \bar{k}_{i t}+\bar{\mu}_{i t} \bar{r}_{t} \bar{k}_{i t}+\bar{\mu}_{i t} \bar{w}_{t}\left(1-\bar{\lambda}_{i t}\right)-\bar{\mu}_{i t} \bar{p}_{t} \bar{c}_{i t}$. We know that $\lim _{t \rightarrow+\infty} \bar{\mu}_{i t} \bar{p}_{t}(1-\delta) \bar{k}_{i t}=0$ and $\lim _{t \rightarrow+\infty} \bar{\mu}_{i t} \bar{r}_{t} \bar{k}_{i t}=0$ (see (40)). We know also that $\lim _{t \rightarrow+\infty} \bar{\mu}_{i t} \bar{w}_{t}\left(1-\bar{\lambda}_{i t}\right)=0$ and $\lim _{t \rightarrow+\infty} \bar{\mu}_{i t} \bar{p}_{t} \bar{c}_{i t}=0$ (see (39)). Therefore, $\lim _{t \rightarrow+\infty} \bar{\mu}_{i t} \bar{p}_{t} \bar{k}_{i t+1}=0$.

Let us eventually prove that an equilibrium exists in the infinite-horizon economy.

Claim $26\left(\overline{\mathbf{p}}, \overline{\mathbf{r}}, \overline{\mathbf{w}},\left(\overline{\mathbf{c}}_{i}, \overline{\mathbf{k}}_{i}, \overline{\boldsymbol{\lambda}}_{i}\right)_{i=1}^{m}, \overline{\mathbf{K}}, \overline{\mathbf{L}}\right)$ is an equilibrium. 
Proof. Consider first the firm. For every truncated $T$-economy, a zero profit condition holds: $\bar{p}_{t}^{T} F\left(\bar{K}_{t}^{T}, \bar{L}_{t}^{T}\right)-\bar{r}_{t}^{T} \bar{K}_{t}^{T}-\bar{w}_{t}^{T} \bar{L}_{t}^{T}=0$. In the limit, for the infinite-horizon economy: $\bar{p}_{t} F\left(\bar{K}_{t}, \bar{L}_{t}\right)-\bar{r}_{t} \bar{K}_{t}-\bar{w}_{t} \bar{L}_{t}=0$, because $\bar{p}_{t}^{T} \rightarrow \bar{p}_{t} \in$ $(0,1), \bar{r}_{t}^{T} \rightarrow \bar{r}_{t} \in(0,1), \bar{w}_{t} \rightarrow \bar{w}_{t} \in(0,1), \bar{K}_{t}^{T}=\sum_{i=1}^{m} \bar{k}_{i t}^{T} \rightarrow \sum_{i=1}^{m} \bar{k}_{i t}=$ $\bar{K}_{t}<+\infty, \bar{L}_{t}^{T}=\sum_{i=1}^{m} \bar{l}_{i t}^{T} \rightarrow \sum_{i=1}^{m} \bar{l}_{i t}=\bar{L}_{t}<+\infty$. If $\left(\bar{K}_{t}, \bar{L}_{t}\right)$ does not maximize the profit in the infinite-horizon economy, then there exists $\left(K_{t}, L_{t}\right)$ such that $\bar{p}_{t} F\left(K_{t}, L_{t}\right)-\bar{r}_{t} K_{t}-\bar{w}_{t} L_{t}>\bar{p}_{t} F\left(\bar{K}_{t}, \bar{L}_{t}\right)-\bar{r}_{t} \bar{K}_{t}-\bar{w}_{t} \bar{L}_{t}=0$ and, so, a critical $\tau$, such that, for any $T>\tau, \bar{p}_{t}^{T} F\left(K_{t}, L_{t}\right)-\bar{r}_{t}^{T} K_{t}-\bar{w}_{t}^{T} L_{t}>$ $\bar{p}_{t}^{T} F\left(\bar{K}_{t}^{T}, \bar{L}_{t}^{T}\right)-\bar{r}_{t}^{T} \bar{K}_{t}^{T}-\bar{w}_{t}^{T} \bar{L}_{t}^{T}=0$ against the fact that $\left(\bar{K}_{t}^{T}, \bar{L}_{t}^{T}\right)$ maximizes the profit in the $T$-economy.

Focus now on consumers. Consider an alternative sequence $\left(\mathbf{c}_{i}, \mathbf{k}_{i}, \boldsymbol{\lambda}_{i}\right)$ which satisfies the budget constraints and the Euler inequalities in the infinite-horizon economy. We have

$$
\begin{aligned}
\Delta_{T} & \equiv \sum_{t=0}^{T} \beta_{i}^{t}\left[u_{i}\left(\bar{c}_{i t}\right)+v_{i}\left(\bar{\lambda}_{i t}\right)\right]-\sum_{t=0}^{T} \beta_{i}^{t}\left[u_{i}\left(c_{i t}\right)+v_{i}\left(\lambda_{i t}\right)\right] \\
& =\sum_{t=0}^{T} \beta_{i}^{t}\left[u_{i}\left(\bar{c}_{i t}\right)-u_{i}\left(c_{i t}\right)\right]+\sum_{t=0}^{T} \beta_{i}^{t}\left[v_{i}\left(\bar{\lambda}_{i t}\right)-v_{i}\left(\lambda_{i t}\right)\right] \\
& \geq \sum_{t=0}^{T} \beta_{i}^{t} u_{i}^{\prime}\left(\bar{c}_{i t}\right)\left(\bar{c}_{i t}-c_{i t}\right)+\sum_{t=0}^{T} \beta_{i}^{t} v_{i}^{\prime}\left(\bar{\lambda}_{i t}\right)\left(\bar{\lambda}_{i t}-\lambda_{i t}\right) \\
& \geq \sum_{t=0}^{T} \bar{\mu}_{i t} \bar{p}_{t}\left(\bar{c}_{i t}-c_{i t}\right)+\sum_{t=0}^{T} \bar{\mu}_{i t} \bar{w}_{t}\left(\bar{\lambda}_{i t}-\lambda_{i t}\right)
\end{aligned}
$$

We observe that

$$
\begin{aligned}
\bar{\mu}_{i t} \bar{p}_{t} \bar{c}_{i t}-\bar{\mu}_{i t} \bar{w}_{t}\left(1-\bar{\lambda}_{i t}\right) & =\bar{\mu}_{i t} \bar{r}_{t} \bar{k}_{i t}+\bar{\mu}_{i t} \bar{p}_{t}(1-\delta) \bar{k}_{i t}-\bar{\mu}_{i t} \bar{p}_{t} \bar{k}_{i t+1} \\
\bar{\mu}_{i t} \bar{p}_{t} c_{i t}-\bar{\mu}_{i t} \bar{w}_{t}\left(1-\lambda_{i t}\right) & \leq \bar{\mu}_{i t} \bar{r}_{t} k_{i t}+\bar{\mu}_{i t} \bar{p}_{t}(1-\delta) k_{i t}-\bar{\mu}_{i t} \bar{p}_{t} k_{i t+1}
\end{aligned}
$$

where the first equality holds because of the Kuhn-Tucker method.

Subtracting member by member, we get

$$
\begin{aligned}
& \bar{\mu}_{i t} \bar{p}_{t}\left(\bar{c}_{i t}-c_{i t}\right)+\bar{\mu}_{i t} \bar{w}_{t}\left(\bar{\lambda}_{i t}-\lambda_{i t}\right) \\
\geq \quad & {\left[\bar{\mu}_{i t} \bar{r}_{t} \bar{k}_{i t}+\bar{\mu}_{i t} \bar{p}_{t}(1-\delta) \bar{k}_{i t}-\bar{\mu}_{i t} \bar{p}_{t} \bar{k}_{i t+1}\right] } \\
& -\left[\bar{\mu}_{i t} \bar{r}_{t} k_{i t}+\bar{\mu}_{i t} \bar{p}_{t}(1-\delta) k_{i t}-\bar{\mu}_{i t} \bar{p}_{t} k_{i t+1}\right]
\end{aligned}
$$

Summing over $t$, we obtain

$$
\begin{aligned}
& \sum_{t=0}^{T} \bar{\mu}_{i t} \bar{p}_{t}\left(\bar{c}_{i t}-c_{i t}\right)+\sum_{t=0}^{T} \bar{\mu}_{i t} \bar{w}_{t}\left(\bar{\lambda}_{i t}-\lambda_{i t}\right) \\
\geq & \sum_{t=0}^{T}\left[\bar{\mu}_{i t} \bar{p}_{t}(1-\delta) \bar{k}_{i t}+\bar{\mu}_{i t} \bar{r}_{t} \bar{k}_{i t}-\bar{\mu}_{i t} \bar{p}_{t} \bar{k}_{i t+1}\right] \\
& -\sum_{t=0}^{T}\left[\bar{\mu}_{i t} \bar{p}_{t}(1-\delta) k_{i t}+\bar{\mu}_{i t} \bar{r}_{t} k_{i t}-\bar{\mu}_{i t} \bar{p}_{t} k_{i t+1}\right]
\end{aligned}
$$


We know also that

$$
\begin{aligned}
& {\left[\bar{\mu}_{i t} \bar{p}_{t}-\bar{\mu}_{i t+1} \bar{p}_{t+1}(1-\delta)-\bar{\mu}_{i t+1} \bar{r}_{t+1}\right] \bar{k}_{i t+1}=0} \\
& {\left[\bar{\mu}_{i t} \bar{p}_{t}-\bar{\mu}_{i t+1} \bar{p}_{t+1}(1-\delta)-\bar{\mu}_{i t+1} \bar{r}_{t+1}\right] k_{i t+1}=0}
\end{aligned}
$$

(point (7) in the Claim 15), that is

$$
\begin{aligned}
\bar{\mu}_{i t} \bar{p}_{t}(1-\delta) \bar{k}_{i t}+\bar{\mu}_{i t} \bar{r}_{t} \bar{k}_{i t} & =\bar{\mu}_{i t-1} \bar{p}_{t-1} \bar{k}_{i t} \\
\bar{\mu}_{i t} \bar{p}_{t}(1-\delta) k_{i t}+\bar{\mu}_{i t} \bar{r}_{t} k_{i t} & =\bar{\mu}_{i t-1} \bar{p}_{t-1} k_{i t}
\end{aligned}
$$

Then,

$$
\begin{aligned}
& \sum_{t=0}^{T} \bar{\mu}_{i t} \bar{p}_{t}\left(\bar{c}_{i t}-c_{i t}\right)+\sum_{t=0}^{T} \bar{\mu}_{i t} \bar{w}_{t}\left(\bar{\lambda}_{i t}-\lambda_{i t}\right) \\
= & \sum_{t=0}^{T}\left(\bar{\mu}_{i t-1} \bar{p}_{t-1} \bar{k}_{i t}-\bar{\mu}_{i t} \bar{p}_{t} \bar{k}_{i t+1}\right)-\sum_{t=0}^{T}\left(\bar{\mu}_{i t-1} \bar{p}_{t-1} k_{i t}-\bar{\mu}_{i t} \bar{p}_{t} k_{i t+1}\right) \\
= & \bar{\mu}_{i,-1} \bar{p}_{-1} k_{i 0}-\bar{\mu}_{i T} \bar{p}_{T} \bar{k}_{i T+1}-\left[\bar{\mu}_{i,-1} \bar{p}_{-1} k_{i 0}-\bar{\mu}_{i T} \bar{p}_{T} k_{i T+1}\right] \\
= & -\bar{\mu}_{i T} \bar{p}_{T} \bar{k}_{i T+1}+\bar{\mu}_{i T} \bar{p}_{T} k_{i T+1} \\
\geq & -\bar{\mu}_{i T} \bar{p}_{T} \bar{k}_{i T+1}
\end{aligned}
$$

Thus, $\lim _{T \rightarrow+\infty} \Delta_{T} \geq 0$ (Claim 25 ) and

$$
\sum_{t=0}^{\infty} \beta_{i}^{t}\left[u_{i}\left(\bar{c}_{i t}\right)+v_{i}\left(\bar{\lambda}_{i t}\right)\right] \geq \sum_{t=0}^{\infty} \beta_{i}^{t}\left[u_{i}\left(c_{i t}\right)+v_{i}\left(\lambda_{i t}\right)\right]
$$

Hence, $\left(\overline{\mathbf{c}}_{i}, \overline{\boldsymbol{\lambda}}_{i}\right)$ maximizes the consumer's objective.

\section{Appendix 3}

Consider a ball of $l^{1}$ with center in the origin and radius $\rho>0: B(0, \rho) \equiv$ $\left\{\mathbf{x} \in l^{1}: \sum_{t=0}^{\infty}\left|x_{t}\right| \leq \rho\right\}$.

Lemma 4 Let $K$ be a subset of $B(0, \rho)$, which satisfies the property: for any $\varepsilon>0$, there exists $\tau$ such that for any $s>\tau$ and for any $\mathbf{x} \in K, \sum_{t=s}^{\infty}\left|x_{t}\right| \leq \varepsilon$. Then $K$ is compact for the $l^{1}$-topology.

Proof. Let $\left(\mathbf{y}^{T}\right)$ be a sequence in $K . B(0, \rho)$ is a compact set for the product topology and contains the sequence $\left(\mathbf{y}^{T}\right)$. Thus, there exists a subsequence $\left(\mathbf{y}^{T_{S}}\right)$ which, for the product topology, converges to some $\mathbf{y} \in B(0, \rho)$.

For any $\varepsilon>0$, there exists $\tau$ such that, for every $s>\tau$ and every $S$, $\sum_{t=s}^{\infty}\left|y_{t}^{T_{S}}\right| \leq \varepsilon$. Take now any $s^{\prime}>s$. We have, for any $s^{\prime}$, any $s>\tau$ and any $S, \sum_{t=s}^{s^{\prime}}\left|y_{t}^{T_{S}}\right| \leq \sum_{t=s}^{\infty}\left|y_{t}^{T_{S}}\right| \leq \varepsilon$. Convergence of $\left(\mathbf{y}^{T_{S}}\right)$ for the product topology implies, for any $s^{\prime}$ and any $s>\tau, \sum_{t=s}^{s^{\prime}}\left|y_{t}\right| \leq \varepsilon$. Thus we get, for any $s>\tau, \sum_{t=s}^{\infty}\left|y_{t}\right| \leq \varepsilon$. Then, $\mathbf{y} \in K$ and $K$ is compact for the $l^{1}$-topology (see the Dunford-Pettis Theorem in Dunford and Schwartz (1966, page 294)). 


\section{Appendix 4}

Let us prove Claim 6

Proof. For any truncated economy, the vector of equilibrium multipliers $\left(\bar{\mu}_{i t}^{T}\right)_{t=0}^{T}$ exists and satisfies $\bar{\mu}_{i t}^{T}=\beta_{i}^{t} u_{i}^{\prime}\left(\bar{c}_{i t}^{T}\right) / \bar{p}_{t}^{T}$ (see point (7) in Claim 15). Since $\lim _{T \rightarrow+\infty} \bar{c}_{i t}^{T}=\bar{c}_{i t} \in(0,+\infty)$ and $\lim _{T \rightarrow+\infty} \bar{p}_{t}^{T}=\bar{p}_{t} \in(0,+\infty)$, we find also

$$
\bar{\mu}_{i t}=\lim _{T \rightarrow+\infty} \frac{\beta_{i}^{t} u_{i}^{\prime}\left(\bar{c}_{i t}^{T}\right)}{\bar{p}_{t}^{T}}=\frac{\beta_{i}^{t} u_{i}^{\prime}\left(\bar{c}_{i t}\right)}{\bar{p}_{t}} \in(0,+\infty)
$$

In addition, we obtain from point (7) in Claim 15:

$$
\begin{aligned}
\bar{\mu}_{i t} \bar{p}_{t} & =\lim _{T \rightarrow+\infty}\left(\bar{\mu}_{i t}^{T} \bar{p}_{t}^{T}\right) \\
& \geq \lim _{T \rightarrow+\infty}\left(\bar{\mu}_{i t+1}^{T}\left[\bar{p}_{t+1}^{T}(1-\delta)+\bar{r}_{t+1}^{T}\right]\right)=\bar{\mu}_{i t+1}\left[\bar{p}_{t+1}(1-\delta)+\bar{r}_{t+1}\right]
\end{aligned}
$$

because $\lim _{T \rightarrow+\infty} \bar{\mu}_{i t}^{T}=\bar{\mu}_{i t} \in(0,+\infty)$.

If $\lim _{T \rightarrow+\infty} \bar{k}_{i t+1}^{T}=\bar{k}_{i t+1}>0$, there is $S$ such that, for any $T>S, \bar{k}_{i t+1}^{T}>0$ and $\bar{\mu}_{i t}^{T} \bar{p}_{t}^{T}=\bar{\mu}_{i t+1}^{T}\left[\bar{p}_{t+1}^{T}(1-\delta)+\bar{r}_{t+1}^{T}\right]$. Thus, $\bar{\mu}_{i t} \bar{p}_{t}=\bar{\mu}_{i t+1}\left[\bar{p}_{t+1}(1-\delta)+\bar{r}_{t+1}\right]$ if $k_{i t+1}>0$.

Summing up, we find that the first-order conditions of point (7) in Claim 15 are satisfied in the limit economy:

$$
\begin{aligned}
& \bar{\mu}_{i t} \bar{p}_{t}=\beta_{i}^{t} u_{i}^{\prime}\left(\bar{c}_{i t}\right) \\
& \bar{\mu}_{i t} \bar{p}_{t} \geq \bar{\mu}_{i t+1} \bar{p}_{t+1}\left(1-\delta+\bar{\rho}_{t+1}\right) \\
& \bar{\mu}_{i t} \bar{p}_{t}=\bar{\mu}_{i t+1} \bar{p}_{t+1}\left(1-\delta+\bar{\rho}_{t+1}\right) \text { if } \bar{k}_{i t+1}>0
\end{aligned}
$$

Let us prove now Proposition 2.

Proof. Assume that such a sequence exists. Let $\omega_{t} \equiv w_{t} / p_{t}$. We have just to show that

$$
\lim _{T \rightarrow+\infty}\left[\sum_{t=0}^{T} \bar{Q}_{t}\left(\bar{C}_{t}-C_{t}\right)+\sum_{t=0}^{T} \bar{Q}_{t} \bar{\omega}_{t}\left(\sum_{i=1}^{m} \bar{\lambda}_{i t}-\sum_{i=1}^{m} \lambda_{i t}\right)\right] \geq 0
$$

a contradiction.

It is enough to prove that feasibility and first-order conditions imply

$$
\left[\sum_{t=0}^{T} \bar{Q}_{t}\left(\bar{C}_{t}-C_{t}\right)+\sum_{t=0}^{T} \bar{Q}_{t} \bar{\omega}_{t}\left(\sum_{i=1}^{m} \bar{\lambda}_{i t}-\sum_{i=1}^{m} \lambda_{i t}\right)\right] \geq-\bar{Q}_{T} \bar{K}_{T+1}
$$

Since capitals are uniformly bounded above, the result follows from $\lim _{T \rightarrow \infty} \bar{Q}_{T}=$ 0 . 
Let us prove inequality (41). Using (11), we find

$$
\begin{aligned}
\Delta_{T} \equiv & \sum_{t=0}^{T} \bar{Q}_{t}\left(\bar{C}_{t}-C_{t}\right)+\sum_{t=0}^{T} \bar{Q}_{t} \bar{\omega}_{t}\left(\sum_{i=1}^{m} \bar{\lambda}_{i t}-\sum_{i=1}^{m} \lambda_{i t}\right) \\
= & \sum_{t=0}^{T} \bar{Q}_{t}\left[F\left(\bar{K}_{t}, \bar{L}_{t}\right)+(1-\delta) \bar{K}_{t}-\bar{K}_{t+1}\right] \\
& -\sum_{t=0}^{T} \bar{Q}_{t}\left[F\left(K_{t}, L_{t}\right)+(1-\delta) K_{t}-K_{t+1}\right] \\
& +\sum_{t=0}^{T} \bar{Q}_{t} \bar{\omega}_{t}\left(m-\bar{L}_{t}\right)-\sum_{t=0}^{T} \bar{Q}_{t} \bar{\omega}_{t}\left(m-L_{t}\right) \\
= & \sum_{t=0}^{T} \bar{Q}_{t}\left[F\left(\bar{K}_{t}, \bar{L}_{t}\right)-F\left(K_{t}, L_{t}\right)+(1-\delta)\left(\bar{K}_{t}-K_{t}\right)-\left(\bar{K}_{t+1}-K_{t+1}\right)\right] \\
& -\sum_{t=0}^{T} \bar{Q}_{t} \bar{\omega}_{t}\left(\bar{L}_{t}-L_{t}\right) \\
\geq & \sum_{t=0}^{T} \bar{Q}_{t}\left[F_{K}\left(\bar{K}_{t}, \bar{L}_{t}\right)\left(\bar{K}_{t}-K_{t}\right)+F_{L}\left(\bar{K}_{t}, \bar{L}_{t}\right)\left(\bar{L}_{t}-L_{t}\right)\right] \\
& +(1-\delta) \sum_{t=0}^{T} \bar{Q}_{t}\left(\bar{K}_{t}-K_{t}\right)-\sum_{t=0}^{T} \bar{Q}_{t}\left(\bar{K}_{t+1}-K_{t+1}\right) \\
& -\sum_{t=0}^{T} \bar{Q}_{t} \bar{\omega}_{t}\left(\bar{L}_{t}-L_{t}\right) \\
= & \sum_{t=0}^{T} \bar{Q}_{t}\left[\bar{\rho}_{t}\left(\bar{K}_{t}-K_{t}\right)+\bar{\omega}_{t}\left(\bar{L}_{t}-L_{t}\right)\right]-\sum_{t=0}^{T} \bar{Q}_{t} \bar{\omega}_{t}\left(\bar{L}_{t}-L_{t}\right) \\
& +(1-\delta) \sum_{t=0}^{T} \bar{Q}_{t}\left(\bar{K}_{t}-K_{t}\right)-\sum_{t=0}^{T} \bar{Q}_{t}\left(\bar{K}_{t+1}-K_{t+1}\right) \\
& \left.\delta+\bar{\rho}_{t}\right)\left(\bar{K}_{t}-K_{t}\right)-\sum_{t=0}^{T} \bar{Q}_{t}\left(\bar{K}_{t+1}-K_{t+1}\right) \\
& \\
& \\
&
\end{aligned}
$$

because $F_{K}\left(\bar{K}_{t}, \bar{L}_{t}\right)=\bar{\rho}_{t}$ and $F_{L}\left(\bar{K}_{t}, \bar{L}_{t}\right)=\bar{\omega}_{t}$ (see points (2) and (3) in Claim 
15). Noticing that $\bar{K}_{0}=K_{0}$ and replacing (7), we get:

$$
\begin{aligned}
\Delta_{T} \geq & \bar{Q}_{0}\left(1-\delta+\bar{\rho}_{0}\right)\left(\bar{K}_{0}-K_{0}\right)+\sum_{t=1}^{T} \bar{Q}_{t}\left(1-\delta+\bar{\rho}_{t}\right)\left(\bar{K}_{t}-K_{t}\right) \\
& -\sum_{t=0}^{T} \bar{Q}_{t}\left(\bar{K}_{t+1}-K_{t+1}\right) \\
= & \sum_{t=0}^{T-1} \bar{Q}_{t+1}\left(1-\delta+\bar{\rho}_{t+1}\right)\left(\bar{K}_{t+1}-K_{t+1}\right) \\
& -\sum_{t=0}^{T-1} \bar{Q}_{t}\left(\bar{K}_{t+1}-K_{t+1}\right)-\bar{Q}_{T}\left(\bar{K}_{T+1}-K_{T+1}\right) \\
\geq & \sum_{t=0}^{T-1}\left[\bar{Q}_{t+1}\left(1-\delta+\bar{\rho}_{t+1}\right)-\bar{Q}_{t}\right]\left(\bar{K}_{t+1}-K_{t+1}\right)-\bar{Q}_{T} \bar{K}_{T+1} \\
= & -\bar{Q}_{T} \bar{K}_{T+1}
\end{aligned}
$$

\section{References}

[1] Becker R.A. (1980). On the long-run steady state in a simple dynamic model of equilibrium with heterogeneous households. Quarterly Journal of Economics 95, 375-382.

[2] Becker R.A. (2005). An example of optimal growth with heterogeneous agents and twisted turnpike. Mimeo, Indiana University.

[3] Becker R.A. (2006). Equilibrium dynamics with many agents. In R.-A. Dana, C. Le Van, T. Mitra and K. Nishimura eds., Handbook of Optimal Growth 1, Springer Verlag, 385-442.

[4] Becker R.A., J.H. Boyd III and C. Foias (1991). The existence of Ramsey equilibrium. Econometrica 59, 441-460.

[5] Becker R.A., R.S. Dubey and T. Mitra (2011). On Ramsey equilibrium: Ownership pattern and inefficiency. Mimeo, Indiana University.

[6] Becker R.A. and C. Foias (1987). A characterization of Ramsey equilibrium. Journal of Economic Theory 41, 173-184.

[7] Becker R.A. and C. Foias (1994). The local bifurcation of Ramsey equilibrium. Economic Theory 4, 719-744.

[8] Becker R.A. and C. Foias (2007). Strategic Ramsey equilibrium dynamics. Journal of Mathematical Economics 43, 318-346. 
[9] Becker R.A. and T. Mitra (2011). Efficient Ramsey equilibria. Macroeconomic Dynamics, electronic publication, forthcoming in print, 2012.

[10] Bergstrom T.C. (1976). How to discard "free disposability" - at no cost. Journal of Mathematical Economics 3, 131-134.

[11] Bewley T. (1982). An integration of equilibrium theory and turnpike theory. Journal of Mathematical Economics 10, 233-267.

[12] Bosi S. and T. Seegmuller (2010). On the Ramsey equilibrium with heterogeneous consumers and endogenous labor supply. Journal of Mathematical Economics 46, 475-492.

[13] Cass D. (1972). On capital overaccumulation in the aggregative, neoclassical model of economic growth: A complete characterization. Journal of Economic Theory 4, 200-233.

[14] Coles J.L. (1985). Equilibrium turnpike theory with constant returns to scale and possibly heterogeneous discount factors. International Economic Review 26 , 671-679.

[15] Coles J.L. (1986). Equilibrium turnpike theory with time-separable utility. Journal of Economic Dynamics and Control 10, 367-394.

[16] Dunford N. and J. Schwartz (1966). Linear Operators, Part 1. Interscience, New York.

[17] Florenzano M. (1999). General equilibrium of financial markets: an introduction. Cahiers de la MSE 1999.76, University of Paris 1.

[18] Florenzano M. and P. Gourdel (1996). Incomplete markets in infinite horizon: debt constraints versus node prices. Mathematical Finance 6, 167-196.

[19] Gale D. and A. Mas-Colell (1975). An equilibrium existence theorem for a general model without ordered preferences. Journal of Mathematical Economics 2, 9-15.

[20] Gale D. and A. Mas-Colell (1979). Corrections to an equilibrium existence theorem for a general model without ordered preferences. Journal of Mathematical Economics 6, 297-298.

[21] Kehoe T.J. (1989). Intertemporal general equilibrium models. In F. Hahn ed., The Economics of Missing Markets, Information, and Games, Clarendon Press, Oxford.

[22] Kehoe T.J. and D.K. Levine (1985). Comparative statics and perfect foresight in infinite horizon economies. Econometrica 53, 433-454.

[23] Kehoe T.J., D.K. Levine and P.M. Romer (1989). Steady states and determinacy of equilibria with infinitely lived agents. In G.R. Feiwel ed., Joan Robinson and Modern Economic Theory, New York University Press, New York. 
[24] Kehoe T.J., D.K. Levine, and P.M. Romer (1990). Determinacy of equilibria in dynamic models with finitely many consumers. Journal of Economic Theory 50, 1-21.

[25] Le Van C., M.H. Nguyen and Y. Vailakis (2007). Equilibrium dynamics in an aggregative model of capital accumulation with heterogeneous agents and elastic labor. Journal of Mathematical Economics 43, 287-317.

[26] Le Van C. and Y. Vailakis (2003). Existence of a competitive equilibrium in a one sector growth model with heterogeneous agents and irreversible investment. Economic Theory 22, 743-771.

[27] Le Van C. and Y. Vailakis (2012). Mimeo, University of Paris 1.

[28] Malinvaud E. (1953). Capital accumulation and efficient allocation of resources. Econometrica 21, 233-268.

[29] Mitra T. (1979). On optimal economic growth with variable discount rates: existence and stability results. International Economic Review 20, 133-145.

[30] Negishi T. (1960). Welfare economics and the existence of equilibrium for a competitive economy. Metroeconomica 23, 92-97.

[31] Rader T. (1971). The Economics of Feudalism. Gordon and Breach, New York.

[32] Rader T. (1972). Theory of General Economic Equilibrium. Academic Press, New York.

[33] Rader T. (1981). Utility over time: The homothetic case. Journal of Economic Theory 25, 219-236.

[34] Ramsey F.P. (1928). A mathematical theory of saving. Economic Journal 38, 543-559.

[35] Sarte P.-D. (1997). Progressive taxation and income inequality in dynamic competitive equilibrium. Journal of Public Economics 66, 145-171.

[36] Sorger G. (1994). On the structure of Ramsey equilibrium: cycles, indeterminacy, and sunspots. Economic Theory 4, 745-764.

[37] Sorger G. (1995). Chaotic Ramsey equilibrium. International Journal of Bifurcation and Chaos 5, 373-380.

[38] Sorger G. (2002). On the long-run distribution of capital in the Ramsey model. Journal of Economic Theory 105, 226-243.

[39] Sorger G. (2005). Differentiated capital and the distribution of wealth. In C. Deissenberg and R.F. Hartl eds., Optimal Control and Dynamic Games, Springer Verlag, 177-196. 
[40] Sorger G. (2008). Strategic saving decisions in the infinite-horizon model. Economic Theory 36, 353-377.

[41] Tirole J. (1990) Intertemporal efficiency, intergenerational transfers, and asset pricing: An introduction. In P. Champsaur et al. eds., Essays in Honor of Edmond Malinvaud, Vol. I: Microeconomics, MIT Press, Cambridge, MA, 116-142. 